\title{
Measurable cardinals and the cofinality of the symmetric group
}

\author{
by \\ Sy-David Friedman and Lyubomyr Zdomskyy (Wien)
}

\begin{abstract}
Assuming the existence of a $P_{2} \kappa$-hypermeasurable cardinal, we construct a model of Set Theory with a measurable cardinal $\kappa$ such that $2^{\kappa}=\kappa^{++}$and the group $\operatorname{Sym}(\kappa)$ of all permutations of $\kappa$ cannot be written as the union of a chain of proper subgroups of length $<\kappa^{++}$. The proof involves iteration of a suitably defined uncountable version of the Miller forcing poset as well as the "tuning fork" argument introduced by the first author and K. Thompson [J. Symbolic Logic 73 (2008)].
\end{abstract}

1. Introduction. A deep theorem of Macpherson and Neumann 16] states that if the symmetric group $\operatorname{Sym}(\kappa)$ consisting of all permutations of a cardinal $\kappa$ can be written as the union of an increasing chain $\left\langle G_{i}: i<\lambda\right\rangle$ of proper subgroups $G_{i}$, then $\lambda>\kappa$. Throughout this paper the minimal $\lambda$ with this property will be denoted by $\operatorname{cf}(\operatorname{Sym}(\kappa))$. It was proven in 22 that for $\kappa=\kappa^{<\kappa}$ the pair $\left(\operatorname{cf}(\operatorname{Sym}(\kappa)), 2^{\kappa}\right)$ can be anything not obviously wrong. More precisely, for every regular $\lambda>\kappa$ and $\theta$ such that $\operatorname{cf}(\theta) \geq \lambda$, there exist a cardinal preserving forcing extension $V^{P}$ such that $\operatorname{cf}(\operatorname{Sym}(\kappa))=\lambda$ and $2^{\kappa}=\theta$ in $V^{P}$. Moreover, for inaccessible $\kappa$ we can assume [17, §1] that $P$ is $\kappa$-directed closed. Therefore if $\kappa$ is supercompact, then it remains so in $V^{Q * P}$, where $Q$ is a Laver preparation forcing making the supercompactness of $\kappa$ indestructible by $\kappa$-directed closed forcing notions. The main result of this paper states that consistency of $\operatorname{cf}(\operatorname{Sym}(\kappa))>\kappa^{+}$at a measurable $\kappa$ can be obtained assuming much less than supercompactness.

THEOREM 1.1. Suppose GCH holds and there exists an elementary embedding $j: V \rightarrow M$ such that $\operatorname{crit}(j)=\kappa$ and $\left(H\left(\kappa^{++}\right)\right)^{V}=\left(H\left(\kappa^{++}\right)\right)^{M}$. Then there exists a forcing extension $V^{\prime}$ of $V$ such that $\kappa$ is still measurable in $V^{\prime}$ and $V^{\prime} \vDash \operatorname{cf}(\operatorname{Sym}(\kappa))=\kappa^{++}$.

2010 Mathematics Subject Classification: Primary 03E35; Secondary 03E55, 03E99.

Key words and phrases: cofinality of the symmetric group, hypermeasurable cardinal, elementary embedding, lifting, Miller forcing, Sacks forcing. 
By work of Gitik [12] a cardinal $\kappa$ of Mitchell order $\kappa^{++}$is required for $\mathrm{GCH}$ to fail at a measurable cardinal; thus the hypothesis of our result is close to optimal (it is in fact equiconsistent with the existence of a cardinal $\kappa$ whose Mitchell order for extenders is $\kappa^{++}+1$ ).

A cardinal $\kappa$ for which there exists an embedding as in Theorem 1.1 will be called $P_{2} \kappa$-hypermeasurable. To the best knowledge of the authors, $\operatorname{cf}(\operatorname{Sym}(\kappa))=\kappa^{+}$for measurable $\kappa$ in all other known models of Set Theory constructed under assumptions weaker than (a certain degree of) supercompactness; see Remark 5.6 for a more detailed discussion.

The idea of the proof of Theorem 1.1 resembles that of the consistency of $\mathfrak{u}<\operatorname{cf}(\operatorname{Sym}(\omega))$ established in [23]. In particular, in Section 2 we introduce a variant of Miller forcing and a (slightly more general than in [14]) variant of Sacks forcing at an inaccessible cardinal $\kappa$. According to Theorem 2.9, iterated forcing constructions where at each stage we take any of these forcing notions do not collapse $\kappa^{+}$. In Section 3 we introduce a new cardinal characteristic $\mathfrak{g}_{c l}(\kappa)$, which is a version for $\kappa$ of the classical groupwise density number $\mathfrak{g}$. Section 4 is devoted to the proof of the fact that suitably arranged iterated forcing constructions considered in Section 2 of length $\kappa^{++}$ make $\operatorname{cf}(\operatorname{Sym}(\kappa))$ equal to $\kappa^{++}$. More precisely, the Miller forcing is responsible for $\operatorname{cf}^{*}(\operatorname{Sym}(\kappa))=\kappa^{++}$, while the Sacks forcing makes $\operatorname{cf}(\operatorname{Sym}(\kappa))$ and $\mathrm{cf}^{*}(\operatorname{Sym}(\kappa))$ equal. (Here $\mathrm{cf}^{*}(\operatorname{Sym}(\kappa))$ is the minimal length of a special chain of proper subgroups of $\operatorname{Sym}(\kappa)$ introduced in Definition 4.1.) And finally, in Section 5 we show how to extend elementary embeddings to forcing extensions considered in Section 2, and thus prove Theorem 1.1. The idea of the proof in Section 5 can be traced back to the work [11], where the "tuning fork" argument was introduced.

2. A variant of Miller forcing for uncountable cardinals. Basic properties. Alternation with Sacks. In this section we suggest one of the possible ways to generalize the Miller forcing introduced in [18] to uncountable cardinals and study some basic properties of iterated forcing constructions, where at each stage we take either the Miller or Sacks forcing poset. The discussion is patterned after Kanamori [14]. It is worth mentioning here that there are other generalizations of Miller forcing (see e.g. [5]).

Throughout this section $\kappa$ denotes a strongly inaccessible cardinal.

Definition 2.1. Let $p \subset \kappa^{<\kappa}$. For $s \in p$ we denote by $C(p, s)$ (or simply by $C(s)$ if $p$ is clear from context) the set $\{\alpha \in \kappa: \hat{s} \alpha \in p\}$.

$\operatorname{Miller}(\kappa)$ denotes the following forcing. A condition is a subset $p$ of $\kappa^{<\kappa}$ such that:

(i) $s \in p, t \subset s \rightarrow t \in p$.

(ii) Each $s \in p$ is increasing and has a proper extension in $p$. 
(iii) For every $\alpha<\kappa$ limit, and $s \in \kappa^{\alpha}$, if $s \uparrow \beta \in p$ for arbitrary large $\beta<\alpha$, then $s \in p$.

(iv) For every $s \in p$ there is $t \in p$ with $s \subset t$ which splits in $p$ (i.e., $C(p, t)$ has more than one element). Moreover, if $t_{0}, t_{1}$ split in $p$ and $t_{0} \subset t_{1}$, then $C\left(p, t_{1}\right) \subset C\left(p, t_{0}\right)$.

(v) If $s \in p$ splits in $p$, then the set $C(p, s)$ is club.

(vi) If $\alpha$ is a limit ordinal, $s \in \kappa^{\alpha}$, and $s\lceil\beta$ splits in $p$ for arbitrary large $\beta<\alpha$, then $s$ splits in $p$ and $C(s)$ is the intersection of $C(s\lceil\beta)$ for all $\beta$ such that $s \uparrow \beta$ splits in $p$.

We order Miller $(\kappa)$ by declaring $p$ to be stronger than $q$ (and write $p \leq q$ ) iff $p \subset q$.

It is clear that $\operatorname{Miller}(\kappa)$ is $\kappa$-closed. For every subtree $p$ of $\kappa^{<\kappa}$ we denote by $\operatorname{Split}(p)$ the family of all $s \in p$ which split in $p$. Given $s \in \kappa^{<\kappa}, \ell(s)$ denotes the length of $s$, i.e. the (unique) $\alpha$ such that $s \in \kappa^{\alpha}$. If $p \in \operatorname{Miller}(\kappa)$ and $\alpha \in \kappa$, then we denote by $\operatorname{Split}_{\alpha}(p)$ the set

$\{s \in p:$ o.t. $(\{t \subsetneq s: t \in \operatorname{Split}(p)\}) \leq \alpha, \forall t \subsetneq s($ o.t. $(s(\ell(t)) \cap C(p, t)) \leq \alpha)\}$.

In what follows we shall heavily apply a fusion argument to Miller $(\kappa)$ as well as to the Sacks forcing.

Definition 2.2. For $q \leq p \in \operatorname{Miller}(\kappa)$ and $\alpha \in \kappa$ the notation $q \leq_{\alpha} p$ means that $\operatorname{Split}_{\alpha}(p)=\operatorname{Split}_{\alpha}(q)$. A sequence $\left\langle p_{\alpha}: \alpha \in \kappa\right\rangle$, where $p_{\alpha} \in$ $\operatorname{Miller}(\kappa)$, is called a fusion sequence if:

(i) If $\beta \leq \alpha$, then $p_{\alpha} \leq p_{\beta}$.

(ii) $p_{\alpha+1} \leq_{\alpha} p_{\alpha}$.

(iii) $p_{\delta}=\bigcap_{\alpha<\delta} p_{\alpha}$ for limit $\delta \in \kappa$.

The following lemma is straightforward.

Lemma 2.3. Let $\left\langle p_{\alpha}: \alpha \in \kappa\right\rangle$ be a fusion sequence. Then $q=\bigcap_{\alpha \in \kappa} p_{\alpha} \in$ $\operatorname{Miller}(\kappa)$ and $q \leq_{\alpha} p_{\alpha}$ for all $\alpha \in \kappa$.

Next, we recall the definition of the Sacks forcing for uncountable cardinals.

Definition 2.4. Let us fix a sequence $\vec{A}=\left\langle A_{\alpha}: \alpha<\kappa\right\rangle$ such that $\left|A_{\alpha}\right|<\kappa$ for all $\alpha$. Let $\mathcal{T}$ be the set of all functions $t$ which satisfy the following conditions:

(i) There exists $\alpha$ such that the domain of $t$ equals $\alpha$.

(ii) For all $\beta \in \operatorname{dom}(t), t(\beta) \in A_{\beta}$.

$\operatorname{Sacks}(\vec{A})$ stands for the forcing whose conditions are subsets $T$ of $\mathcal{T}$ such that: 
(1) $s \in T, t \subset s \rightarrow t \in T$.

(2) Each $t$ has a proper extension in $T$.

(3) If $t \in \mathcal{T}$ and the set of such $\beta$ that $t \uparrow \beta \in T$ is unbounded in $\ell(t)$, then $t \in T$.

(4) There exists a club $C(T)$ such that the set $\operatorname{succ}_{T}(t)$ of immediate successors of an element $t \in T$ with domain $\alpha$ coincides with $\left\{t^{\wedge} a\right.$ : $\left.a \in A_{\alpha}\right\}$ provided $\alpha \in C(T)$, and $\left|\operatorname{succ}_{T}(t)\right|=1$ otherwise.

Extension is defined by $S \leq T$ iff $S$ is a subset of $T$.

When each $A_{\alpha}$ is $\{0,1\}$ we get the usual Sacks forcing considered in [7, 11, 14]. Some other sequences $\vec{A}$ are employed in [9]. Yet another sequence will be used in Section 4. But the basic properties (e.g. chain condition, fusion) of $\operatorname{Sacks}(\vec{A})$ do not really depend on $\vec{A}$.

Given any $T \in \operatorname{Sacks}(\vec{A})$ and $i \in \kappa$, we denote by $\operatorname{Split}_{i}(T)$ the set $\left\{t \in T:(\exists j \leq i) \ell(t)=\alpha_{j}\right\}$, where $\left\langle\alpha_{i}: i \in \kappa\right\rangle$ is the increasing enumeration of $C(T)$. Now the notions of $\leq_{\alpha}$ and of a fusion sequence can be introduced for $\operatorname{Sacks}(\vec{A})$ in the same way as for $\operatorname{Miller}(\kappa)$.

If $\gamma$ is an ordinal and $S^{0}, S^{1}$ are disjoint subsets of $\gamma$ such that $S^{0} \cup S^{1}=\gamma$, then we denote by $S T_{S^{0}, S^{1}, \vec{A}}$ the forcing poset $\mathbb{P}_{\gamma}$ from the iterated forcing construction $\left\langle\mathbb{P}_{\xi}, \dot{\mathbb{Q}}_{\eta}: \xi \leq \gamma, \eta<\gamma\right\rangle$ with supports of size $\leq \kappa$ defined as follows:

$$
\left\{\eta<\gamma: \mathbb{P}_{\eta} \Vdash \dot{\mathbb{Q}}_{\eta}=\operatorname{Miller}(\kappa)\right\}=S^{0},\left\{\eta<\gamma: \mathbb{P}_{\eta} \Vdash \dot{\mathbb{Q}}_{\eta}=\operatorname{Sacks}(\vec{A})\right\}=S^{1} .
$$

Definition 2.5. Suppose that $\alpha \leq \kappa$ and $\left\langle p_{\beta}: \beta \in \alpha\right\rangle$ is a decreasing sequence of elements of $S T_{S^{0}, S^{1}, \vec{A}}$. The "meet" $q=\bigwedge_{\beta \in \alpha} p_{\beta} \in S T_{S^{0}, S^{1}, \vec{A}}$ is defined as follows: $\operatorname{supp}(q) \stackrel{,}{=} \bigcup_{\beta \in \alpha} \operatorname{supp}\left(p_{\beta}\right)$ and for every $\xi \in \operatorname{supp}(q)$, $q\left\lceil\xi \Vdash q(\xi)=\bigcap_{\beta \in \alpha} p_{\beta}(\xi)\right.$. (Note that in case $\alpha=\kappa$ there could be no such q.)

In order to prove that $\kappa^{+}$is preserved by $S T_{S^{0}, S^{1}, \vec{A}}$ and $\kappa^{++}$is preserved for $\gamma=\kappa^{++}$we need to employ a suitable variant of fusion.

Definition 2.6. Suppose that $\alpha \in \kappa, F \in[\gamma]^{<\kappa}$, and $q, p \in S T_{S^{0}, S^{1}, \vec{A}}$. $q \leq_{F, \alpha} p$ means that $q \leq p$ and $q\left\lceil\xi \Vdash q(\xi) \leq_{\alpha} p(\xi)\right.$ for all $\xi \in F\left[\left(^{1}\right)\right.$.

A sequence $\left\langle\left(p_{\alpha}, F_{\alpha}\right): \alpha \in \kappa\right\rangle$ is a generalized fusion sequence (for $\left.S T_{S^{0}, S^{1}, \vec{A}}\right)$ iff:

(i) $\left|F_{\alpha}\right|<\kappa$ for all $\alpha \in \kappa$.

(ii) $F_{\alpha} \supset F_{\beta}$ for all $\beta \leq \alpha<\kappa$.

(iii) $p_{\alpha+1} \leq_{F_{\alpha}, \alpha} p_{\alpha}$ for all $\alpha$.

(iv) If $\delta$ is limit, then $F_{\delta}=\bigcup_{\beta<\alpha} F_{\beta}$ and $p_{\delta}=\bigwedge_{\alpha<\delta} p_{\alpha}$.

(v) $\bigcup\left\{F_{\alpha}: \alpha \in \kappa\right\}=\bigcup\left\{\operatorname{supp}\left(p_{\alpha}\right): \alpha<\kappa\right\}$.

$\left({ }^{1}\right)$ The preorder $\leq_{\alpha}$ here depends on whether $\xi \in S^{0}$ or $\xi \in S^{1}$. 
The easy but technical proof of the following lemma is left to the reader.

Lemma 2.7. Let $\left\langle\left(p_{\alpha}, F_{\alpha}\right): \alpha \in \kappa\right\rangle$ be a generalized fusion sequence for $S T_{S^{0}, S^{1}, \vec{A}}$. Then $q=\bigwedge_{\alpha<\kappa} p_{\alpha} \in S T_{S^{0}, S^{1}, \vec{A}}$ and $q \leq_{F_{\alpha}, \alpha} p_{\alpha}$ for all $\alpha \in \kappa$.

There is no loss of generality to assume that each $A_{\alpha}$ is an element of $\kappa$.

Definition 2.8. Suppose that $p \in S T_{S^{0}, S^{1}, \vec{A}}, F \subset \operatorname{supp}(p)$ with $|F|<\kappa$, and $\sigma: F \rightarrow \kappa^{<\kappa}$. Then $p \mid \sigma$ is a function with the same domain as $p$ such that $(p \mid \sigma)(\xi)$ equals $p(\xi)$ if $\xi \notin F$ and $p(\xi)_{\sigma(\xi)}$ otherwise, where for $q \in \operatorname{Miller}(\kappa) \cup \operatorname{Sacks}(\vec{A})$ and $t \in \kappa^{<\kappa}, q_{t}$ denotes the set of all elements of $q$ compatible with $t$.

It is clear that $p \mid \sigma \in S T_{S^{0}, S^{1}, \vec{A}}$ if and only if for every $\xi \in F$ we have $(p \mid \sigma) \mid \xi \Vdash_{\xi} \sigma(\xi) \in p(\xi)$. If $p \mid \sigma \in S T_{S^{0}, S^{1}, \vec{A}}$, then we say that $\sigma$ lies on $p$.

TheOREM 2.9. For every ordinal $\gamma$ and decomposition $\gamma=S^{0} \cup S^{1}$ the forcing $S T_{S^{0}, S^{1}, \vec{A}}$ preserves cardinals $\leq \kappa^{+}$.

Suppose that $2^{\kappa}=\kappa^{+}$in $V$. If $\gamma<\kappa^{++}$, then there exists a dense subset $W_{\gamma} \subset S T_{S^{0}, S^{1}, \vec{A}}$ of size $\left|W_{\gamma}\right| \leq \kappa^{+}$. If $\gamma=\kappa^{++}$, then $S T_{S^{0}, S^{1}, \vec{A}}$ has the $\kappa^{++}$-chain condition.

Similar results were discussed in [14] and [7] for the Sacks forcing. Nevertheless, we give complete proofs here. Our exposition follows [14]. The first part of Theorem 2.9 follows from the lemma below.

LEMMA 2.10.

(1) Assume that $p \in S T_{S^{0}, S^{1}, \vec{A}}$ and $p \Vdash \dot{z} \in V$. Then for every $F \in[\gamma]^{<\kappa}$ and $\alpha_{0} \in \kappa$ there exist $q \leq_{F, \alpha_{0}} p$ and $x \in V$ with $|x| \leq \kappa$ such that $q \Vdash \dot{z} \in x$.

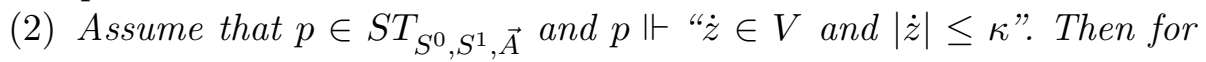
every $F \in[\gamma]^{<\kappa}$ and $\alpha_{0} \in \kappa$ there exist $q \leq_{F, \alpha_{0}} p$ and $x \in V$ with $|x| \leq \kappa$ such that $q \Vdash \dot{z} \subset x$.

Proof. It is well-known how to obtain the second item from the first one (see [14, Theorem 2.3]).

In order to prove the first item we shall inductively construct a generalized fusion sequence $\left\langle\left(p_{\alpha}, F_{\alpha}\right): \alpha \in \kappa\right\rangle$ with $\left(p_{\beta}, F_{\beta}\right)=(p, F)$ for all $\beta \leq \alpha_{0}$, and $x \in V$ of size $|x| \leq \kappa$ such that $q=\bigwedge_{\alpha \in \kappa} p_{\alpha}$ and $x$ are as required. The trivial description of how to construct $F_{\alpha}$ 's is omitted. The limit step of the construction is obvious, so we concentrate on the successor case.

Let us enumerate as $\left\{\sigma_{\alpha, i}: i \in \eta\right\}$ all ground model functions $\sigma: F_{\alpha} \rightarrow$ $\kappa^{\alpha+1}$ which lie on some $r \leq p_{\alpha}$ so that $r=r|\sigma, r| \xi \Vdash \sigma(\xi) \mid \alpha \in \operatorname{Split}_{\alpha}\left(p_{\alpha}(\xi)\right)$ for all $\xi \in F_{\alpha}$, and $\sigma(\xi)(\alpha)=\alpha$ for all $\xi \in F_{\alpha} \cap S^{0}$. (Here $\eta<\kappa$ is a cardinal.) We shall construct a sequence $\left\langle p_{\alpha, i}: i \in \eta\right\rangle$ as follows. Set $p_{\alpha,-1}=p_{\alpha}$ and suppose that we have already constructed a decreasing sequence $\left\langle p_{\alpha, j}: j<i\right\rangle$ 
such that $p_{\alpha, j} \leq_{F_{\alpha}, \alpha} p_{\alpha, k}$ for all $k \leq j<i$. If $i$ is limit, we set $p_{\alpha, i}=\bigwedge_{j<i} p_{\alpha, j}$. Suppose that $i=j+1$. If there is no $r \leq p_{\alpha, j}$ such that $r=r \mid \sigma_{\alpha, j}$ and $r\left\lceil\xi \Vdash \sigma(\xi)\left\lceil\alpha \in \operatorname{Split}_{\alpha}\left(p_{\alpha}(\xi)\right)\right.\right.$ for all $\xi \in F_{\alpha}$, we set $p_{\alpha, i}=p_{\alpha, j}$. And if there is such an $r$, let $r_{\alpha, j} \leq r$ and $x_{\alpha, j} \in V$ be such that $r_{\alpha, j} \Vdash \dot{z}=x_{\alpha, j}$. Now, using the Maximal Principle we define $p_{\alpha, j+1}$ to be the amalgamation of $p_{\alpha, j}$ and $r_{\alpha, j}$ as in the proof of [14, Theorem 2.2]. More precisely:

(a) $\operatorname{supp}\left(p_{\alpha, j+1}\right)=\operatorname{supp}\left(r_{\alpha, j}\right)$.

(b) If $\xi \in F_{\alpha}$, then $p_{\alpha, j+1}(\xi)$ is such that

$$
r_{\alpha, j} \mid \xi \Vdash p_{\alpha, j+1}(\xi)=\left(p_{\alpha, j}(\xi) \backslash p_{\alpha, j}(\xi)_{\sigma_{\alpha, j}(\xi)}\right) \cup r_{\alpha, j}(\xi),
$$

and for any condition $c \leq p_{\alpha, j+1}\left\lceil\xi\right.$ incompatible with $r_{\alpha, j}\lceil\xi$,

$$
c \Vdash_{\xi} p_{\alpha, j+1}(\xi)=p_{\alpha, j}(\xi) .
$$

(c) If $\xi \notin F_{\alpha}$, then $p_{\alpha, j+1}(\xi)$ is such that

$$
r_{\alpha, j} \mid \xi \Vdash p_{\alpha, j+1}(\xi)=r_{\alpha, j}(\xi),
$$

and for any condition $c \leq p_{\alpha, j+1}\left\lceil\xi\right.$ incompatible with $r_{\alpha, j}\lceil\xi$,

$$
c \Vdash_{\xi} p_{\alpha, j+1}(\xi)=p_{\alpha, j}(\xi) .
$$

Now we let $p_{\alpha+1}=\bigwedge_{i \in \eta} p_{\alpha, i}$. It follows that $p_{\alpha+1} \leq_{F_{\alpha}, \alpha} p_{\alpha}$. This completes our construction of $\left\langle\left(p_{\alpha}, F_{\alpha}\right): \alpha \in \kappa\right\rangle$. Set $x=\left\{x_{\alpha, i}\right\}$.

Claim 2.11. Suppose that $r \leq q$. Then there exists a sequence $\left\langle r_{\alpha}\right.$ : $\alpha \in \kappa\rangle$ of elements of $S T_{S^{0}, S^{1}, \vec{A}}$ with $r_{0}=r$, a sequence $\left\langle\sigma_{\alpha}: F_{\alpha} \rightarrow \kappa^{<\kappa}\right|$ $\alpha<\kappa\rangle$, and sequences $\left\langle\mu_{\alpha, \xi}, \nu_{\alpha, \xi}: \alpha \in \kappa, \xi \in F_{\alpha}\right\rangle$ of ordinals less than $\kappa$ such that:

(i) If $\beta<\alpha$, then $r_{\alpha} \leq r_{\beta}$.

(ii) If $\xi \in F_{\alpha}$, then $\ell\left(\sigma_{\alpha}(\xi)\right)=\mu_{\alpha, \xi}+1$.

(iii) If $\beta<\alpha$, then $\sigma_{\beta}(\xi) \subsetneq \sigma_{\alpha}(\xi)$ for all $\xi \in F_{\beta}$.

(iv) For every $\xi \in F_{\alpha+1}$ we have $r_{\alpha+1}\left\lceil\xi \Vdash " r_{\alpha+1}(\xi)=r_{\alpha+1}(\xi)_{\sigma_{\alpha+1}(\xi)}\right.$, $\sigma_{\alpha+1}(\xi)\left\lceil\mu_{\alpha+1, \xi}\right.$ splits in $r_{\alpha}(\xi)$, and $\sigma_{\alpha+1}(\xi)\left\lceil\mu_{\alpha+1, \xi} \in \operatorname{Split}_{\nu_{\alpha+1, \xi}}(q(\xi))\right.$ ".

(v) If $\delta$ is limit, then:

- $\sigma_{\delta}(\xi)\left\lceil\mu_{\delta, \xi}=\bigcup_{\alpha<\delta} \sigma_{\alpha}(\xi)\right.$.

- $\sigma_{\delta}(\xi)\left(\mu_{\delta, \xi}\right)=\sup \left\{\sigma_{\alpha}(\xi)\left(\mu_{\alpha, \xi}\right): \alpha<\delta\right\}$ for all $\xi \in F_{\delta} \cap S^{0}$ (we assume that $\sigma_{\alpha}(\xi)=\emptyset$ for all $\left.\xi \notin F_{\alpha}\right)$.

- $\nu_{\delta, \xi}=\sup _{\alpha<\delta} \nu_{\alpha, \xi}$ for all $\xi \in F_{\delta}$.

- $r_{\delta}\left\lceil\xi \Vdash " \sigma_{\delta}(\xi) \in r_{\delta}(\xi), \sigma_{\delta}(\xi)\left\lceil\mu_{\delta, \xi}\right.\right.$ splits in $r_{\delta}(\xi)$, and $\sigma_{\delta}(\xi)\left\lceil\mu_{\delta, \xi} \in \operatorname{Split}_{\nu_{\delta, \xi}}(q(\xi))\right.$ " for all $\xi \in F_{\delta}$.

Proof. The construction proceeds by induction. For limit $\delta$ we simply set $\sigma_{\delta}(\xi)$ and $\nu_{\delta, \xi}$ to be as required in (v) and $r_{\delta}=\bigwedge_{\alpha<\delta} r_{\alpha}$. Thus $\mu_{\delta, \xi}=$ $\sup _{\alpha<\delta} \mu_{\alpha, \xi}$. Let us fix any $\alpha<\delta$ and $\xi \in F_{\alpha} \cap S^{0}$. From the above 
it follows that $r_{\delta}\left\lceil\xi \Vdash\right.$ " $\sigma_{\beta}(\xi)\left\lceil\mu_{\beta, \xi}\right.$ splits in $r_{\alpha}(\xi)$ for all $\alpha<\beta<\delta$ ", and hence $r_{\delta}\left\lceil\xi \Vdash\right.$ " $\sigma_{\delta}(\xi)\left\lceil\mu_{\delta, \xi}\right.$ splits in $r_{\alpha}(\xi)$ ", and consequently $r_{\delta}\lceil\xi \Vdash$ " $\sigma_{\delta}(\xi)\left\lceil\mu_{\delta, \xi}\right.$ splits in $r_{\delta}(\xi)=\bigcap_{\beta<\delta} r_{\beta}(\xi)$ ". By the definition of $\operatorname{Miller}(\kappa)$, $r_{\delta}\left\lceil\xi \Vdash C\left(r_{\alpha}(\xi), \sigma_{\delta}(\xi)\left\lceil\mu_{\delta, \xi}\right)=\bigcap_{\alpha<\beta<\delta} C\left(r_{\alpha}(\xi), \sigma_{\beta}(\xi)\left\lceil\mu_{\beta, \xi}\right)\right.\right.\right.$, and hence $r_{\delta}\lceil\xi \Vdash$ $\sigma_{\delta}(\xi)\left(\mu_{\delta, \xi}\right)=\sup _{\alpha<\beta<\delta} \sigma_{\beta}(\xi)\left(\mu_{\beta, \xi}\right) \in C\left(r_{\alpha}(\xi), \sigma_{\delta}(\xi)\left\lceil\mu_{\delta, \xi}\right)\right)$, which implies that $r_{\delta} \backslash \xi \Vdash \sigma_{\delta}(\xi)\left(\mu_{\delta, \xi}\right) \in C\left(r_{\delta}(\xi), \sigma_{\delta}(\xi)\left\lceil\mu_{\delta, \xi}\right)=\bigcap_{\alpha<\delta} C\left(r_{\alpha}(\xi), \sigma_{\delta}(\xi)\left\lceil\mu_{\delta, \xi}\right)\right)\right.$, which gives $r_{\delta}\left\lceil\xi \Vdash \sigma_{\delta}(\xi) \in r_{\delta}(\xi)\right.$. Finally, the equalities $\sigma_{\delta}(\xi)\left\lceil\mu_{\delta, \xi}=\right.$ $\bigcup_{\alpha<\delta} \sigma_{\alpha}(\xi)\left\lceil\mu_{\alpha, \xi}\right.$ and $\nu_{\delta, \xi}=\sup _{\alpha<\xi} \nu_{\alpha, \xi}$ combined with $r_{\delta}\left\lceil\xi \Vdash \sigma_{\alpha}(\xi)\left\lceil\mu_{\alpha, \xi} \in\right.\right.$ $\operatorname{Split}_{\nu_{\alpha, \xi}}(q(\xi))$ imply $r_{\delta}\left\lceil\xi \Vdash \sigma_{\delta}(\xi)\left\lceil\mu_{\delta, \xi} \in\right.\right.$ Split $_{\nu_{\delta, \xi}}(q(\xi))$, which completes the limit step.

At successor step $\alpha+1$ consider the increasing enumeration $\left\langle\xi_{i}: i\langle\eta\rangle\right.$ of $F_{\alpha+1}$ and find a decreasing sequence $\left\langle u_{i}: i<\eta\right\rangle$ of elements of $S T_{S^{0}, S^{1}, \vec{A}}$ as follows: Set $u_{i}=\bigwedge_{j<i} u_{j}$ for limit $i$. Now given $u_{i}$, find $v \leq u_{i} \mid \xi_{i}, \pi \in \kappa^{\mu+1}$ for some $\mu \in \kappa$, and $\nu \in \kappa$ such that $\pi \supset \sigma_{\alpha}\left(\xi_{i}\right)$ if $\xi_{i} \in F_{\alpha}$ and

$$
v \Vdash_{\xi_{i}} \pi \in r_{\alpha}\left(\xi_{i}\right), \pi\left\lceil\mu \in \operatorname{Split}_{\nu}\left(q\left(\xi_{i}\right)\right) \cap \operatorname{Split}\left(r_{\alpha}\left(\xi_{i}\right)\right) .\right.
$$

Then we set

$$
u_{i+1}=\widehat{v^{-}} r_{\alpha}\left(\xi_{i}\right)_{\pi} \widehat{ } r_{\alpha}\left\lceil\left(\gamma \backslash\left(\xi_{i}+1\right)\right), \quad \sigma_{\alpha+1}\left(\xi_{i}\right)=\pi .\right.
$$

( $\mu_{\alpha+1, \xi_{i}}$ and $\nu_{\alpha+1, \xi_{i}}$ automatically become equal to $\mu$ and $\nu$ respectively.) With $u_{i}$ 's thus defined, we set $r_{\alpha+1}=\bigwedge_{i<\eta} u_{i}$. This completes the inductive construction, hence the proof of the claim.

The following claim is obvious.

Claim 2.12. There exists a club $C \subset \kappa$ such that $\mu_{\alpha, \xi}=\nu_{\alpha, \xi}=\alpha$ and $\sigma_{\alpha}(\xi)\left(\mu_{\alpha, \xi}\right)=\alpha$ for every $\alpha \in C$ and $\xi \in F_{\alpha}$. Consequently, $r_{\alpha}\lceil\xi \Vdash$ $\sigma_{\alpha}(\xi)\left\lceil\alpha \in \operatorname{Split}_{\alpha}(q(\xi))\right.$ for every $\alpha \in C$ and $\xi \in F_{\alpha}$.

We are in a position now to finish the proof of Lemma 2.10, Let $C$ be as in Claim 2.12 and $\alpha \in C$. Then $\sigma_{\alpha}=\sigma_{\alpha, i}$ for some $i<\eta$ (see the construction of $p_{\alpha+1}$ at the beginning of the proof of Lemma 2.10). Since $r_{\alpha+1} \leq q \leq p_{\alpha, i}$, Claim 2.11(iv) implies that for every $\xi \in F_{\alpha+1} \supset F_{\alpha}$ we have $r_{\alpha+1} \mid \xi \Vdash r_{\alpha+1}(\xi)=r_{\alpha+1}(\xi)_{\sigma_{\alpha}(\xi)}$. Therefore the construction of $p_{\alpha, i+1}$ is nontrivial. Since $r_{\alpha+1} \leq q \leq p_{\alpha, i+1}, r_{\alpha+1}=r_{\alpha+1}\left|\sigma_{\alpha} \leq p_{\alpha, i+1}\right| \sigma_{\alpha, i}=r_{\alpha, i}$, and hence $r_{\alpha+1} \Vdash \dot{z}=x_{\alpha, i}$. Therefore for every $r \leq q$ there exists $r^{\prime} \leq r$ such that $r^{\prime} \Vdash \dot{z} \in x$, which finishes our proof.

Proof of Theorem 2.9. The proof is analogous to that of [14, Lemma 3.1]. Let $W_{\gamma}$ be the set of those $q \in S T_{S^{0}, S^{1}, \vec{A}}$ such that:

(i) There is an increasing sequence $\left\langle F_{\alpha}: \alpha \in \kappa\right\rangle$ of subsets of $\gamma$ such that $\left|F_{\alpha}\right|<\kappa$ for all $\alpha, F_{\delta}=\bigcup_{\alpha \in \delta} F_{\alpha}$ for limit $\delta$, and $\bigcup_{\alpha \in \kappa} F_{\alpha}=\operatorname{supp}(q)$.

(ii) For every $\alpha$ there exists a (possibly empty) collection $\Sigma_{\alpha}$ of ground model functions $\sigma: F_{\alpha} \rightarrow \kappa^{\alpha+1}$ of size $\left|\Sigma_{\alpha}\right|<\kappa$ such that $q \mid \sigma \in$ 
$S T_{S^{0}, S^{1}, \vec{A}}$ for all $\sigma \in \bigcup_{\alpha \in \kappa} \Sigma_{\alpha}$, and whenever $\beta \in \kappa$ and $r \leq q$, there exist $\alpha>\beta$ and $\sigma \in \Sigma_{\alpha}$ such that $r$ and $q \mid \sigma$ are compatible.

The proof of Lemma 2.10 gives that $W_{\gamma}$ is dense in $S T_{S^{0}, S^{1}, \vec{A}}$. In addition, almost literal repetition of the proof of [14, Lemma 3.1] shows that if a pair of sequences

$$
\left\langle\left\langle F_{\alpha}: \alpha \in \kappa\right\rangle,\left\langle\Sigma_{\alpha}: \alpha \in \kappa\right\rangle\right\rangle
$$

is a witness for $q_{i} \in W_{\gamma}, i \in 2$, then $q_{0} \leq q_{1} \leq q_{0}$ in $S T_{S^{0}, S^{1}, \vec{A}}$. It suffices to note that there are at most $\kappa^{+}$-many such pairs.

Finally, the fact that $S T_{S^{0}, S^{1}, \vec{A}}$ has $\kappa^{++}$-chain condition provided $\gamma=$ $\kappa^{++}$is a direct consequence of [1, Theorem 2.2].

At this point we would like to note that there has been extensive work by Eisworth, Rosłanowski, Shelah and perhaps others on possible generalizations of proper forcing to uncountable cardinals (see, e.g., [8, 19]). It is plausible that Theorem 2.9 follows from one of the general results about uncountable versions of proper forcing. However, Claim 2.11 will play a central role in the proof of Claim 5.4, and for this reason we gave a complete proof of Theorem 2.9 instead of trying to put it into the framework of the results from [8] or [19].

3. $\operatorname{Miller}(\kappa)$ and a variant of the groupwise density number. Throughout this section $\kappa$ is strongly inaccessible, $2^{\kappa}=\kappa^{+}$in $V, \kappa^{++}=$ $S^{0} \sqcup S^{1}, \vec{A}=\left\langle A_{\alpha}: \alpha \in \kappa\right\rangle$ is a sequence of ordinals below $\kappa$, and $S^{0}$ is $\kappa^{+}$-stationary (we use $\sqcup$ as notation for disjoint union). Here we define a new cardinal characteristic of $\kappa$ and show that iteration of $\operatorname{Miller}(\kappa)$ pushes it to $\kappa^{++}$.

Definition 3.1. We say that $\mathcal{G} \subset[\kappa]^{\kappa}$ is a cgd-family (abbreviated from club groupwise dense) if for every continuous increasing function $\phi: \kappa \rightarrow \kappa$ there exists a club $C \subset \kappa$ such that $\bigcup_{\alpha \in C} \phi(\alpha+1) \backslash \phi(\alpha) \in \mathcal{G}$, and for every $A \in \mathcal{G}$ and $B \in[\kappa]^{\kappa}$ such that $|B \backslash A|<\kappa$ we have $B \in \mathcal{G}$. In what follows, the minimal size of a collection $\mathfrak{G}$ of cgd-families with empty intersection is denoted by $\mathfrak{g}_{c l}(\kappa)$.

Theorem 3.2. Suppose that $G$ is an $S T_{S^{0}, S^{1}, \vec{A}^{-}}$-generic filter. Then $V[G]$ $\models \mathfrak{g}_{c l}(\kappa)=\kappa^{++}$.

The proof of Theorem 3.2 is divided into a sequence of lemmas.

Lemma 3.3. Suppose that $G$ is an $S T_{S^{0}, S^{1}, \vec{A}^{-}}$generic filter. Then for every subset $x$ of $\kappa$ such that $x \in V[G]$ there exists $\gamma<\kappa^{++}$such that $x \in V\left[G_{\gamma}\right]$, and the smallest such $\gamma$ has cofinality $\leq \kappa$. 


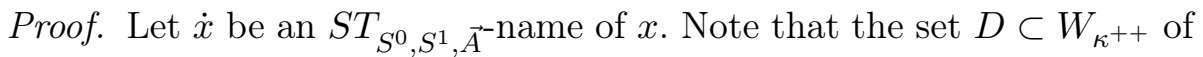
all $q \in S T_{S^{0}, S^{1}, \vec{A}}$ as in the proof of Theorem 2.9 with the additional property that for every $\sigma \in \Sigma_{\alpha}$ the condition $q \mid \sigma$ decides $\dot{x}(\beta)$ for all $\beta<\alpha$, is dense in $S T_{S^{0}, S^{1}, \vec{A}}$. (Any $q$ obtained along the lines of the proof of Lemma 2.10 with an extra requirement that $r_{\alpha, j}$ decides $\dot{x}(\beta)$ for all $\beta<\alpha$ belongs to $D$.) Item (ii) from the proof of Theorem 2.9 implies that $\left\{q \mid \sigma: \sigma \in \bigcup_{\alpha \in \kappa} \Sigma_{\alpha}\right\}$ is predense below $q$. Therefore for every $q \in D$ and $\beta \in \kappa$ there exists a subset $E_{q, \beta}$ predense below $q$ of size $\leq \kappa$ and such that each element of $E_{q, \beta}$ decides $\dot{x}(\beta)$. From the above it follows that for every $q \in D$ we have $q \Vdash \dot{x}=\pi$, where $\pi=\left\{\left\langle\left\langle\beta, \check{i}_{\beta, r}\right\rangle, r\right\rangle: \beta \in \kappa, r \in E_{q, \beta}\right\}$ and $r \Vdash \dot{x}(\beta)=i_{\beta, r}$. The rest of the proof is straightforward.

The following lemma resembles [3, Lemma 5.10].

LEMmA 3.4. Let $G$ be an $S T_{S^{0}, S^{1}, \vec{A}^{-}}$eneric filter and $\mathcal{F} \in V[G]$ be a cgd-family. There is a $\kappa^{+}$-closed unbounded set of ordinals $\eta<\kappa^{++}$for which $\mathcal{F} \cap V\left[G_{\eta}\right] \in V\left[G_{\eta}\right]$ and $\mathcal{F} \cap V\left[G_{\eta}\right]$ is a cgd-family in $V\left[G_{\eta}\right]$.

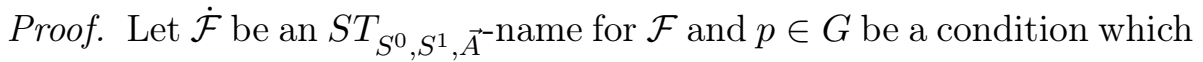
forces that $\dot{\mathcal{F}}$ is a cgd-family, and $\gamma<\kappa^{++}$be such that $p \in \mathbb{P}_{\gamma}$. The proof of Lemma 3.3 yields a set $\Pi_{\gamma}$ of $\mathbb{P}_{\gamma}$-names of size $\left|\Pi_{\gamma}\right|=\kappa^{+}$such that for every

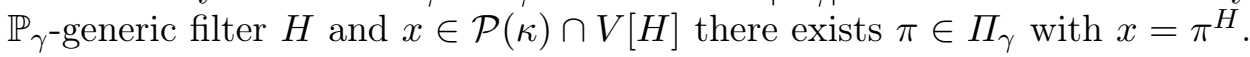
For every $\pi \in \Pi_{\gamma}$ we denote by $B(\pi)$ a maximal antichain of conditions in $\mathbb{P}_{\kappa^{++}}$that decide whether $\pi \in \dot{\mathcal{F}}$. Let $\eta_{1}=\eta_{1}(\gamma)$ be the supremum of the union of the supports of all conditions appearing in some $B(\pi), \pi \in \Pi_{\gamma}$. (Recall that $S T_{S^{0}, S^{1}, \vec{A}}$ has $\kappa^{++}$-c.c.) Then $\mathcal{F} \cap V\left[G_{\gamma}\right] \in V\left[G_{\eta_{1}}\right]$.

For every $\pi \in \Pi_{\gamma}$ we can find a maximal antichain $A(\pi)$ below $p$ whose elements decide whether $\pi$ is (the range of) a continuous increasing function, and if $q \in A(\pi)$ decides that $\pi$ is such, then for some $\xi(\pi, q)>\gamma$ and $\theta_{\pi, q} \in \Pi_{\xi(\pi, q)}, q$ forces $\theta_{\pi, q}$ to be a club and $\bigcup_{\alpha \in \theta_{\pi, q}}[\pi(\alpha), \pi(\alpha+1)) \in \dot{\mathcal{F}}$. Let $\eta_{2}$ be the upper bound of the set

$$
\left\{\xi(\pi, q): \pi \in \Pi_{\gamma}, q \in \bigcup_{\pi \in \Pi_{\gamma}} A(\pi)\right\} \cup\left\{\operatorname{supp}(q): q \in \bigcup_{\pi \in \Pi_{\gamma}} A(\pi)\right\} .
$$

Then $\eta(\gamma):=\max \left\{\eta_{1}, \eta_{2}\right\}$ has the properties $\dot{\mathcal{F}}^{H} \cap V\left[H_{\gamma}\right] \in V\left[H_{\eta(\gamma)}\right]$, and if $\psi \in V\left[H_{\gamma}\right]$ is any continuous increasing sequence, then there is a club $C \in$ $V\left[H_{\eta(\gamma)}\right]$ such that $\bigcup_{\alpha \in C}[\psi(\alpha), \psi(\alpha+1)) \in \dot{\mathcal{F}}^{H}$, where $H$ is any $S T_{S^{0}, S^{1}, \vec{A}^{-}}$ generic filter containing $p$.

Let $E \subset \kappa^{++}$be the $\kappa^{+}$-closed unbounded set of those $\eta$ such that $\eta(\gamma) \leq$ $\eta$ for all $\gamma<\eta$. We claim that $E$ is as required. Indeed, by Lemma 3.3 for every $\eta \in E$ we have $\mathcal{F} \cap V\left[G_{\eta}\right]=\left\{\pi^{G_{\eta}}: \exists \gamma<\eta\left(\pi \in \Pi_{\gamma} \wedge B(\pi) \cap G_{\eta} \neq \emptyset \wedge\right.\right.$ the unique element of $B(\pi) \cap G_{\eta}$ forces $\left.\left.\pi \in \dot{\mathcal{F}}\right)\right\}$, and the last set is obviously in $V\left[G_{\eta}\right]$. Now suppose that $\psi \in V\left[G_{\eta}\right]$ is a continuous increasing function 
from $\kappa$ to $\kappa$. Applying Lemma 3.3 we can find $\gamma<\eta$ such that $\psi \in V\left[G_{\gamma}\right]$. From the above it follows that there is a club $C \in V\left[G_{\eta(\gamma)}\right] \subset V\left[G_{\eta}\right]$ such that $\bigcup_{\alpha \in C}[\psi(\alpha), \psi(\alpha+1)) \in \dot{\mathcal{F}}^{G} \cap V\left[G_{\eta}\right]$, which finishes our proof.

Lemma 3.5. For every $p \in \operatorname{Miller}(\kappa)$ there exists a continuous increasing sequence $\left\langle\nu_{\alpha}: \alpha \in \kappa\right\rangle$ such that for every club $C$ there exists $q \leq p$ such that the range of every branch through $q$ is almost (= modulo a subset of size $<\kappa)$ contained in $\bigcup_{\alpha \in C}\left[\nu_{\alpha}, \nu_{\alpha+1}\right)$.

Proof. We define the desired sequence $\left\langle\nu_{\alpha}\right\rangle_{\alpha \in \kappa}$ inductively. Choose $\nu_{0}$ arbitrary. For limit $\delta \in \kappa$ we set $\nu_{\delta}=\sup _{\alpha \in \delta} \nu_{\alpha}$. After $\nu_{\alpha}$ is defined, let $\beta>\nu_{\alpha}$ be such that for every $s \in p$ whose range is a subset of $\nu_{\alpha}$ and $\xi \in\left[\nu_{\alpha}, \beta\right)$, if $s^{\wedge} \xi \in p$, then the range of the smallest extension $t \in \operatorname{Split}(p)$ of $s^{\wedge} \xi$ is contained in $\beta$. We set $\nu_{\alpha+1}=\beta$.

We claim that the sequence $\left\langle\nu_{\alpha}: \alpha \in \kappa\right\rangle$ is as required. Indeed, it is continuous by the construction. Let $C$ and $D \subset \bigcup_{\alpha \in C}\left[\nu_{\alpha}, \nu_{\alpha+1}\right)$ be clubs. (The role of $D$ here is to ensure that the splitting nodes of the condition $q$ constructed below split into clubs rather than into sets containing clubs. We could take, e.g., $D=\left\{\alpha \in C: \nu_{\alpha}=\alpha\right\}$.) Let $q$ be the tree generated by the set of those $s=s_{1}^{\wedge} \xi \in p$ such that $s_{1} \in \operatorname{Split}(p)$ and for every $t \leq s$, if $t \in \operatorname{Split}(p)$, then $s(\ell(t)) \in \bigcup_{\alpha \in C \backslash \mu(t)}\left[\nu_{\alpha}, \nu_{\alpha+1}\right) \cap D$, where $\mu(t)$ is the minimal ordinal $\mu$ such that $\nu_{\mu}$ contains the range of $t$. Then $q \in \operatorname{Miller}(\kappa)$. It suffices to note that the range of each branch through $q$ is a subset of $\bigcup_{\alpha \in C}\left[\nu_{\alpha}, \nu_{\alpha+1}\right) \cup \beta$, where $\beta$ is the range of the stem (= smallest splitting element) of $p$.

Proof of Theorem 3.2. A simple density argument based on Lemma 3.5 shows that if $H$ is a Miller $(\kappa)$-generic filter and $\mathcal{F}$ is a cgd-family in $V$, then the range of $\bigcap H \in \kappa^{\kappa}$ is almost included in some $F \in \mathcal{F}$.

Now let $\mathfrak{F} \in V[G]$ be a collection of cgd-families of size $\kappa^{+}$. For every $\mathcal{F} \in \mathfrak{F}$ Lemma 3.4 yields a $\kappa^{+}$-closed unbounded set $C_{\mathcal{F}} \in \mathcal{P}\left(\kappa^{++}\right) \cap V$ such that $\mathcal{F} \cap V\left[G_{\eta}\right] \in V\left[G_{\eta}\right]$ and $\mathcal{F} \cap V\left[G_{\eta}\right]$ is a cgd-family in $V\left[G_{\eta}\right]$ for every $\eta \in C_{\mathcal{F}}$. Let us fix $\eta \in S^{0} \cap \bigcap_{\mathcal{F} \in \mathfrak{F}} C_{\mathcal{F}}$. From the above it follows that $G_{\eta+1}=G_{\eta} * H$, where $H$ is a $\operatorname{Miller}(\kappa)$-generic filter over $V\left[G_{\eta}\right]$. As we already noted, $H$ gives rise to a subset $X \in V\left[G_{\eta+1}\right]$ of $\kappa$ such that for every $\mathcal{F} \in \mathfrak{F}$ there exists $F \in \mathcal{F} \cap V\left[G_{\eta}\right]$ such that $X \subset F$. Therefore $X \in \bigcap \mathfrak{F}$, which finishes our proof.

\section{A new lower bound for the cofinality of the symmetric group.} In this section $\kappa$ denotes a strongly inaccessible cardinal. The main result of this section says that for a certain sequence $\vec{A}$, if both $S^{0}$ and $S^{1}$ are $\kappa^{+}$-stationary and $G$ is $S T_{S^{0}, S^{1}, \vec{A}^{-}}$generic, then $V[G] \vDash \operatorname{cf}(\operatorname{Sym}(\kappa))=\kappa^{++}$. The motivation for this is given in Section 5 . We follow the strategy of the 
proof of [23, Theorem 2.2]. In its turn that proof relies upon the methods developed in $[21, \S 2]$.

Following [23] we give the following definition.

Definition 4.1. For a subset $A$ of $\kappa$ we shall identify the group $\operatorname{Sym}(A)$ with the subgroup of $\operatorname{Sym}(\kappa)$ consisting of permutations $\sigma$ such that $\sigma\lceil(\kappa \backslash A)$ $=\operatorname{id}_{\kappa \backslash A}$.

For every increasing $\psi \in \kappa^{\kappa}$ let $P_{\psi}$ be the group $\prod_{\alpha \in \kappa} \operatorname{Sym}(\psi(\alpha+1)$ $\backslash \psi(\alpha))$, which will be identified with a subgroup of $\operatorname{Sym}(\kappa)$. Let $\mathrm{cf}^{*}(\operatorname{Sym}(\kappa))$ be the least cardinal $\lambda$ such that it is possible to express $\operatorname{Sym}(\kappa)=\bigcup_{i<\lambda} \Gamma_{i}$ as the union of a chain of proper subgroups such that for every increasing continuous $\phi \in \kappa^{\kappa}$ there exists $i \in \lambda$ such that $P_{\phi}$ is a subgroup of $\Gamma_{i}$.

For an increasing function $\theta: \kappa \rightarrow \kappa$ we set $\tilde{\theta}(\alpha)=\sup _{\xi \in \alpha} \theta(\xi)$ and $Q_{\theta}=P_{\tilde{\theta}}$. (Note that $\tilde{\theta}$ is continuous and $P_{\theta} \subset Q_{\theta}$.)

The following lemma resembles [23, Theorem 2.6]. But the proofs of Lemma 4.2 and of Theorem 2.6 from [23] are completely different.

Lemma 4.2. $\operatorname{cf}^{*}(\operatorname{Sym}(\kappa)) \geq \mathfrak{g}_{c l}(\kappa)$.

Proof. The proof is divided into two steps.

Claim 4.3. For every $\pi \in \operatorname{Sym}(\kappa)$ there exists a continuous increasing $\psi \in \kappa^{\kappa}$ such that $\pi \in P_{\psi}$.

Proof. For any $\alpha \in \kappa$ we set $\beta(\alpha)=\min \{\pi(\xi): \xi \geq \alpha\}$ and $\gamma(\alpha)=$ $\sup \{\pi(\xi): \xi \in \alpha\}$. Since $\pi$ is a bijection, Fodor's lemma implies that $\beta(\alpha) \geq \alpha$ for club many $\alpha$ 's. Therefore there exists a club $C \subset \kappa$ such that $\gamma\left\lceil C=\operatorname{id}_{C}\right.$ and $\beta(\alpha) \geq \alpha$ for all $\alpha \in C$. Now, the increasing bijective enumeration $\psi: \kappa \rightarrow C \cup\{0\}$ is as required.

Given any $B \in[\kappa]^{\kappa}$, we denote by $e_{B}: \kappa \rightarrow B$ the increasing bijective enumeration of $B$. Note that continuous strictly increasing functions from $\kappa$ to $\kappa$ are exactly those of the form $e_{C}$ for a club $C$.

Claim 4.4. Let $\Gamma$ be a subgroup of $\operatorname{Sym}(\kappa)$ containing $\operatorname{Sym}_{0}(\kappa)=\{\pi$ : $\pi(\alpha)=\alpha$ for all but $<\kappa$ many $\alpha$ 's $\}$ and such that $\langle\Gamma, g\rangle \neq \operatorname{Sym}(\kappa)$ for all $g \in \operatorname{Sym}(\kappa)$, and $\mathcal{G}_{\Gamma}=\left\{A \in[\kappa]^{\kappa}: \forall B\left(|B \backslash A|<\kappa \rightarrow Q_{e_{B}} \not \subset \Gamma\right)\right\}$. Then $\mathcal{G}_{\Gamma}$ is a cgd-family.

Proof. Let $\phi: \kappa \rightarrow \kappa$ be a continuous increasing function. Since $\operatorname{Sym}_{0}(\kappa)$ $\subset \Gamma$, the family $\mathcal{G}_{\Gamma}$ is closed under modifications of size $<\kappa$ of its elements. Thus it is enough to show that there exists a club $C$ such that, letting $C_{\phi}=\bigcup_{\alpha \in C}[\phi(\alpha), \phi(\alpha+1))$, we have $C_{\phi} \in \mathcal{G}_{\Gamma}$, which means $Q_{e_{C_{\phi}}}$ $\not \subset \Gamma$. Assume to the contrary that $Q_{e_{C_{\phi}}} \subset \Gamma$ for every club $C \subset \kappa$. Set $O=\bigcup_{\alpha \text { odd }}[\phi(\alpha), \phi(\alpha+1))$. We claim that $\operatorname{Sym}(O) \subset \Gamma$. Once this is established, we get a contradiction with [16, Lemma 2.4]. Let us fix 
$\sigma \in \operatorname{Sym}(O)$. Claim 4.3 yields a continuous increasing $\psi: \kappa \rightarrow \kappa$ such that $\sigma \in \prod_{\xi \in \kappa} \operatorname{Sym}\left(\left[e_{O} \circ \psi(\xi), e_{O} \circ \psi(\xi+1)\right) \cap O\right)$. Set $C=\{\alpha: \alpha$ is limit and $\left.\phi(\alpha)=\sup _{\xi \in \alpha} e_{O} \circ \psi(\xi)\right\}$. It is clear that $C$ is club. Since elements of $C$ are limit ordinals, the choice of $O$ ensures that $C_{\phi} \cap O=\emptyset$. We claim that

$$
[\phi(\alpha), \phi(\alpha+1)) \cap\left[e_{O} \circ \psi(\xi), e_{O} \circ \psi(\xi+1)\right)=\emptyset
$$

for all $\alpha \in C$ and $\xi \in \kappa$. Indeed, if $\xi<\alpha$, then $e_{O} \circ \psi(\xi+1)<\sup _{\eta<\alpha} e_{O} \circ \psi(\eta)$ $=\phi(\alpha)$. Now suppose $\xi \geq \alpha$. Then $O \ni e_{O} \circ \psi(\xi) \geq \phi(\alpha)$, and therefore $[\phi(\alpha), \phi(\alpha+1)) \cap O=\emptyset$ implies $e_{O} \circ \psi(\xi) \geq \phi(\alpha+1)$, which proves $(1)$. For any $\xi \in \kappa$ consider $\alpha(\xi), \beta(\xi) \in \kappa$ such that $\alpha(\xi)=\min \{\alpha \in C: \phi(\alpha) \geq$ $\left.e_{O} \circ \psi(\xi+1)\right\}$ and $\phi(\alpha(\xi))$ is the $\beta(\xi)$ th element of $C_{\phi}$. Equation (1) gives

$$
\begin{aligned}
{\left[e_{O} \circ \psi(\xi), e_{O} \circ \psi(\xi+1)\right) } & \subset\left[\sup _{\beta<\beta(\xi)} e_{C_{\phi}}(\beta), e_{C_{\phi}}(\beta(\xi))\right) \\
& =\left[\tilde{e}_{C_{\phi}}(\beta(\xi)), \tilde{e}_{C_{\phi}}(\beta(\xi)+1)\right),
\end{aligned}
$$

and therefore

$$
\prod_{\xi \in \kappa} \operatorname{Sym}\left(\left[e_{O} \circ \psi(\xi), e_{O} \circ \psi(\xi+1)\right) \cap O\right) \subset Q_{e_{C_{\phi}}} \subset \Gamma,
$$

which implies $\sigma \in \Gamma$ and thus completes our proof.

Let us express $\operatorname{Sym}(\kappa)=\bigcup_{i<\lambda} \Gamma_{i}$ as the union of an increasing chain of proper subgroups such that each $P_{\psi}$ is contained in some $\Gamma_{i}$. Since $\left|\operatorname{Sym}_{0}(\kappa)\right|$ $=\kappa$ and $\lambda>\kappa$, we can assume that $\operatorname{Sym}_{0}(\kappa) \subset \Gamma_{0}$. For every $A \in[\kappa]^{\kappa}$ there exists $i \in \lambda$ such that $Q_{e_{A}}=P_{\tilde{e_{A}}} \subset \Gamma_{i}$, consequently $\bigcap_{i \in \lambda} \mathcal{G}_{\Gamma_{i}}=\emptyset$, and therefore $\mathfrak{g}_{c l}(\kappa) \leq \lambda$, which finishes our proof. Wemma 4.2

Definition 4.5. Let $\phi_{0}: \kappa \rightarrow \kappa$ be the continuous increasing function such that $\phi_{0}(0)=\omega$ and $\phi_{0}(\alpha+1)=\phi_{0}(\alpha)+\alpha$ for all $\alpha \in \kappa$. We set $N_{\alpha}=\operatorname{Sym}\left(\phi_{0}(\alpha+1) \backslash \phi_{0}(\alpha)\right), \vec{N}=\left\langle N_{\alpha}: \alpha<\kappa\right\rangle$, and $S T_{S^{0}, S^{1}}=S T_{S^{0}, S^{1}, \vec{N}}$.

Each branch $\vec{t}=\langle t(\alpha)\rangle_{\alpha \in \kappa}$ of $T \in \operatorname{Sacks}(\vec{N})$ can be naturally identified with an element of $\sigma_{\vec{t}} \in P_{\phi_{0}}$ such that $\sigma_{\vec{t}} \uparrow\left(\phi_{0}(\alpha+1) \backslash \phi_{0}(\alpha)\right)=t(\alpha)$. We also need the following

Definition 4.6. $[\kappa]^{\kappa, \kappa}$ denotes the set $\{A \subset \kappa:|A|=|\kappa \backslash A|=\kappa\}$. If $A \in[\kappa]^{\kappa, \kappa}$ and $\sigma \in \operatorname{Sym}(\kappa)$, then $\sigma^{A}$ is defined by $\sigma^{A}\left(e_{A}(\alpha)\right)=e_{A}(\sigma(\alpha))$. If $\Gamma$ is a subgroup of $\operatorname{Sym}(\kappa)$, then $\Gamma^{A}=\left\{\sigma^{A}: \sigma \in \Gamma\right\}$ and $\Gamma(A)=\{\sigma\lceil A$ : $\sigma \in \Gamma, \sigma[A]=A\}$.

The next lemma is of crucial importance for the proof of the equality $\operatorname{cf}^{*}(\operatorname{Sym}(\kappa))=\operatorname{cf}(\operatorname{Sym}(\kappa))$ in $V^{S T_{S^{0}, S^{1}}}$ for $\kappa^{+}$-stationary subsets $S^{0}, S^{1}$ of $\kappa^{++}$.

Lemma 4.7. Let $\psi: \kappa \rightarrow \kappa$ be a continuous increasing function. Then for every $T \in \operatorname{Sacks}(\vec{N})$ there exists $A \in[\kappa]^{\kappa, \kappa}$ such that for every $\pi \in P_{\psi}$ there exists $S \leq T$ such that $\sigma_{\vec{s}} \mid A=\pi^{A}$ for all branches $\vec{s}$ of $S$. 
Proof. Let $B=\left\{\alpha \in \kappa: \psi(\alpha)=\phi_{0}(\alpha)=\alpha\right\}$. Then $B$ is obviously a club. Consider $D \in[C(T) \cap B]^{\kappa}$ such that $D$ is a discrete subspace of $\kappa$ and find a club $C^{\prime} \subset C(T) \backslash D$. Set $A=\bigcup_{\alpha \in D}\left(\phi_{0}(\alpha+1) \backslash \phi_{0}(\alpha)\right)=\bigcup_{\alpha \in D}[\alpha, \alpha+\alpha)$. We claim that for every $\beta<\kappa$ there exists $\alpha(\beta) \in D$ such that

$$
e_{A}[\psi(\beta), \psi(\beta+1)) \subset\left[\phi_{0}(\alpha(\beta)), \phi_{0}(\alpha(\beta)+1)\right)=[\alpha(\beta), \alpha(\beta) \cdot 2) .
$$

Indeed, let us fix $\beta$ and find $\alpha(\beta)$ such that $e_{A}(\psi(\beta)) \in[\alpha(\beta), \alpha(\beta) \cdot 2)$. Since $\alpha(\beta)$ is a fixed point of $\phi_{0}$, it is indecomposable, i.e. it is not equal to the sum of any two smaller ordinals. By our choice of $D, \alpha(\beta)>\sup (D \cap \alpha(\beta))$, and hence o.t. $\left(A \cap \phi_{0}(\alpha(\beta))\right)<\phi_{0}(\alpha(\beta))=\alpha(\beta)$. Therefore o.t. $(A \cap \alpha(\beta) \cdot 2)=$ o.t. $\left(A \cap \phi_{0}(\alpha(\beta))\right)+\alpha(\beta)=\alpha(\beta)$, which means that $e_{A}(\alpha(\beta))=\alpha(\beta) \cdot 2$. It follows that $\psi(\beta)=$ o.t. $\left(A \cap e_{A}(\psi(\beta))\right)<$ o.t. $(A \cap \alpha(\beta) \cdot 2)=\alpha(\beta)$. Since $\alpha(\beta)$ is a fixed point of $\psi$, we conclude that $\psi(\beta+1)<\alpha(\beta)$. In other words, o.t. $\left(A \cap e_{A}(\psi(\beta+1))\right)<$ o.t. $\left(A \cap \phi_{0}(\alpha(\beta)+1)\right)$, which implies the inequality $e_{A}(\psi(\beta+1))<\phi_{0}(\alpha(\beta)+1)$ and thus proves $(2)$.

Now, let us fix $\pi \in P_{\psi}$. A direct verification shows that $S \in \operatorname{Sacks}(\vec{N})$ such that $C(S)=C^{\prime}$ and for every $\beta \in \kappa$ and $s \in S$ we have

$$
e_{A}^{-1} \circ s(\alpha(\beta)) \circ e_{A}\lceil[\psi(\beta), \psi(\beta+1))=\pi \uparrow[\psi(\beta), \psi(\beta+1))
$$

is as required.

Lemma 4.8. Suppose that $\lambda=\operatorname{cf}(\operatorname{Sym}(\kappa))<\mathrm{cf}^{*}(\operatorname{Sym}(\kappa))$ and $\left\langle\Gamma_{i}: i \in \lambda\right\rangle$ is an increasing chain of proper subgroups of $\operatorname{Sym}(\kappa)$ such that $\operatorname{Sym}(\kappa)=$ $\bigcup_{i<\lambda} \Gamma_{i}$. Then there exists a continuous increasing $\psi: \kappa \rightarrow \kappa$ such that $P_{\psi}^{A} \not \subset \Gamma_{i}(A)$ for all $i<\lambda$ and $A \in[\kappa]^{\kappa, \kappa}$.

Proof. Let us fix $A_{0} \in[\kappa]^{\kappa, \kappa}$. The same argument as in the proof of [4, Lemma 2.7] gives us a continuous increasing $\psi: \kappa \rightarrow \kappa$ such that $P_{\psi}^{A_{0}} \not \subset$ $\Gamma_{i}\left(A_{0}\right)$ for all $i<\lambda$. We claim that this $\psi$ is as required. Indeed, let $A \in[\kappa]^{\kappa, \kappa}$ and $\pi \in \operatorname{Sym}(\kappa)$ be such that $\pi\lceil A$ is the monotone bijection between $A$ and $A_{0}$, and $j \in \lambda$ be such that $\pi \in \Gamma_{j}$. It is easy to check that if $P_{\psi}^{A} \subset \Gamma_{i}(A)$ for some $i$, then $P_{\psi}^{A_{0}} \subset \Gamma_{\max \{i, j\}}\left(A_{0}\right)$, which contradicts our choice of $\psi$.

The next lemma can be proven by the same methods as Lemma 3.4 .

Lemma 4.9. Suppose that $2^{\kappa}=\kappa^{+}$in $V, \kappa^{++}=S^{0} \cup S^{1}$ is a decomposition into two $\kappa^{+}$-stationary subsets, and $G$ is an $S T_{S^{0}, S^{1}}$-generic filter. For every $\Pi \subset \operatorname{Sym}(\kappa)$ of size $|\Pi| \leq \kappa^{+}$and every sequence $\left\langle\Gamma_{i}: i<\kappa^{+}\right\rangle \in$ $V[G]$ of subgroups of Sym $(\kappa)$ there is a $\kappa^{+}$-closed unbounded set of ordinals $\eta<\kappa^{++}$for which $\Pi \in V\left[G_{\eta}\right],\left\langle\Gamma_{i} \cap V\left[G_{\eta}\right]: i<\kappa^{+}\right\rangle \in V\left[G_{\eta}\right]$, and for every $A \in[\kappa]^{\kappa, \kappa} \cap V\left[G_{\eta}\right]$ and $i<\kappa^{+}$we have $\Gamma_{i}(A) \cap V\left[G_{\eta}\right]=\left(\Gamma_{i} \cap V\left[G_{\eta}\right]\right)(A)$.

Finally, we are in a position to prove the following theorem, which is the main result of this section. 
Theorem 4.10. Let $S^{0}, S^{1}$, and $G$ be as in Lemma 4.9. Then $V[G] \vDash$ $\operatorname{cf}(\operatorname{Sym}(\kappa))=\kappa^{++}$.

Proof. Suppose to the contrary that $V[G] \vDash \operatorname{Sym}(\kappa)=\kappa^{+}$. Let $\left\langle\Gamma_{i}\right.$ : $i\left\langle\kappa^{+}\right\rangle \in V[G]$ be an increasing chain of subgroups of $\operatorname{Sym}(\kappa)$ such that $\operatorname{Sym}(\kappa)=\bigcup_{i<\kappa^{+}} \Gamma_{i}$. By Theorem 3.2 and Lemma 4.2 we have $V[G] \vDash$ $\operatorname{cf}^{*}(\operatorname{Sym}(\kappa))=\kappa^{++}$. Lemma 4.8 yields a continuous increasing $\psi: \kappa \rightarrow \kappa$ such that for every $A \in[\kappa]^{\kappa, \kappa}$ and $i<\kappa^{+}$we have $P_{\psi}^{A} \not \subset \Gamma_{i}(A)$. Fix $A_{*} \in[\kappa]^{\kappa, \kappa}$ and for every $i<\kappa^{+}$find $\pi_{i} \in P_{\psi}$ such that $\pi_{i}^{A_{*}} \in P_{\psi}^{A_{*}} \backslash \Gamma_{i}\left(A_{*}\right)$. Observe that $\Pi^{A} \not \subset \Gamma_{i}(A)$ for any $A \in[\kappa]^{\kappa, \kappa}$ and $i<\kappa^{+}$, where $\Pi=\left\{\pi_{i}\right.$ : $\left.i<\kappa^{+}\right\}$. (The condition $\pi_{i}^{A} \notin \Gamma_{i}(A)$ holds at least starting from $i$ such that $\Gamma_{i}$ contains an extension of the order-preserving bijection between $A_{*}$ and $A$.)

Let $\eta<\kappa^{++}$be an element of the $\kappa^{+}$-closed unbounded subset provided by Lemma 4.9 for $\left\langle\Gamma_{i}: i<\kappa^{+}\right\rangle$and $\Pi$ for which $\dot{\mathbb{Q}}_{\eta}=\operatorname{Sacks}(\vec{N})$, i.e. $\eta \in S^{1}$. We can additionally require $A_{*} \in V\left[G_{\eta}\right]$. Suppose that $H$ is the $\operatorname{Sacks}(\vec{N})$ generic filter over $V\left[G_{\eta}\right]$ such that $G_{\eta+1}=G_{\eta} * H$ and $\vec{h}$ is the common branch of all trees in $H$. Applying Lemma 4.7 we conclude that the set

$$
\begin{aligned}
&\left\{S \in \operatorname{Sacks}(\vec{N}): \exists A \in[\kappa]^{\kappa, \kappa} \cap V\left[G_{\eta}\right] \exists \pi \in \Pi\right. \\
&\left(\pi^{A} \notin\left(\Gamma_{i} \cap V\left[G_{\eta}\right]\right)(A) \wedge S \Vdash \sigma_{\vec{h}}\left\lceil A=\pi^{A}\right)\right\}
\end{aligned}
$$

is dense for all $i<\kappa^{+}$. Therefore for every $i$ there exist $A_{i} \in[\kappa]^{\kappa, \kappa} \cap V\left[G_{\eta}\right]$ and $j(i)<\kappa^{+}$such that $\sigma=\sigma_{\vec{h}}\left\lceil A_{i}=\pi_{j(i)}^{A_{i}} \notin\left(\Gamma_{i} \cap V\left[G_{\eta}\right]\right)\left(A_{i}\right)\right.$. Let $i<\kappa^{+}$ be such that $\sigma \in \Gamma_{i}$. Then

$$
\left(\Gamma_{i} \cap V\left[G_{\eta}\right]\right)\left(A_{i}\right) \not \supset \pi_{j(i)}^{A_{i}}=\sigma\left\lceil A_{i} \in \Gamma_{i}\left(A_{i}\right) \cap V\left[G_{\eta}\right],\right.
$$

which contradicts our choice of $\eta$.

Now it is natural to ask whether we needed to employ $\operatorname{Sacks}(\vec{N})$ at all.

Question 4.11. Is $\operatorname{cf}(\operatorname{Sym}(\kappa)) \geq \mathfrak{g}_{c l}(\kappa)$ ?

The cardinal characteristic $\mathfrak{g}_{c l}(\kappa)$ seems to be a natural generalization of the classical groupwise density number $\mathfrak{g}$ introduced in [2] and it was proved in [4] that $\operatorname{cf}(\operatorname{Sym}(\omega)) \geq \mathfrak{g}$. But the methods of [4] do not seem to be applicable to Question 4.11 .

5. Proof of Theorem 1.1. Without loss of generality, $j=j_{E}$ for some $\left(\kappa, \kappa^{++}\right.$)-extender $E$ (such embeddings will be called $\left(\kappa, \kappa^{++}\right)$-extender $u l$ trapowers in what follows) so that $M=\{j(f)(a): f \in V, f: H(\kappa) \rightarrow V$, and $\left.a \in H\left(\kappa^{++}\right)\right\}$(see, e.g, [13, pp. 381-384] $\left({ }^{2}\right)$.

$\left(^{2}\right)$ What we actually use here is the following analogue of [13, Lemma 20.30]: A cardinal $\kappa$ is $P_{2} \kappa$-hypermeasurable iff there exists a $\left(\kappa, \kappa^{++}\right)$-extender $E$ such that $H\left(\kappa^{++}\right) \subset \mathrm{Ult}_{E}$ and $\kappa^{++}<j_{E}(\kappa)$. 
Claim 5.1. There exists a cardinal preserving forcing extension $V^{\prime}$ of $V$ such that $G C H$ holds in $V^{\prime}$ and $j$ can be extended to an elementary embedding $j^{\prime}: V^{\prime} \rightarrow M^{\prime}$ satisfying the following conditions:

(i) $H\left(\kappa^{++}\right)^{V^{\prime}}=H\left(\kappa^{++}\right)^{M^{\prime}}$.

(ii) $j^{\prime}$ is given by a $\left(\kappa, \kappa^{++}\right)$-extender ultrapower so that $M^{\prime}=\left\{j^{\prime}(f)(a)\right.$ : $f \in V^{\prime}, f: H(\kappa)^{V^{\prime}} \rightarrow V^{\prime}$, and $\left.a \in H\left(\kappa^{++}\right)^{V^{\prime}}\right\}\left({ }^{3}\right)$.

(iii) There exist disjoint $\kappa^{+}$-stationary (in $V^{\prime}$, and hence in $\left.M^{\prime}\right)$ subsets $S^{0}, S^{1} \in M^{\prime}$ of $\kappa^{++}$such that $S^{0} \cup S^{1}=\kappa^{++}$, and a sequence $\left\langle\left(S_{k}^{0}, S_{k}^{1}\right): k \in \kappa\right\rangle$, where $S_{k}^{0}$ and $S_{k}^{1}$ are disjoint $\rho_{k}^{+}$-stationary subsets of $\rho_{k}^{++}$for which $\rho_{k}^{++}=S_{k}^{0} \cup S_{k}^{1}$, such that $j^{\prime}\left\langle\left(S_{k}^{0}, S_{k}^{1}\right)\right.$ : $k \in \kappa\rangle(\kappa)=\left(S^{0}, S^{1}\right)$. (Here $\rho_{k}$ denotes the $k$ th inaccessible cardinal below $\kappa, k<\kappa$.)

Proof. We define a forcing poset $\mathbb{R}$ as follows. Let $\mathbb{R}_{0}=\left\{\mathbf{1}_{0}\right\}$. For $k \leq \kappa$ we denote by $\dot{\mathbb{S}}_{k}$ an $\mathbb{R}_{k}$-name for the poset $F n\left(\rho_{k}^{++}, 2, \rho_{k}^{++}\right)$adding one Cohen subset to $\rho_{k}^{++}$(see [15]). Proceeding this way along all inaccessible cardinals $\leq \kappa$ and using reverse Easton supports we define $\mathbb{R}$. Let $G$ be an $\mathbb{R}_{\kappa}$-generic over $V, g$ be an $\mathbb{S}_{\kappa}=\dot{\mathbb{S}}_{\kappa}^{G}$-generic over $V[G], G_{k}=G \cap \mathbb{R}_{k}$ and $g_{k}$ be such that $G_{k+1}=G_{k} * g_{k}$ for all $k<\kappa$. Note that $g_{k}$ is the characteristic function of some subset of $S_{k}^{0}$ of $\rho^{++}$. It is clear that $S_{k}^{0}$ as well as its complement meet all subsets of $\rho_{k}^{++}$of size $\rho_{k}^{++}$which appear in $V\left[G_{k}\right]$. Since $\mathbb{R}_{k+1}$ has $\rho_{k}^{++}$-c.c., each $\rho_{k}^{+}$-closed unbounded (in $\rho_{k}^{++}$) subset $C^{\prime} \in V\left[G_{k+1}\right]$ contains a $\rho_{k}^{+}$-closed unbounded (in $\rho_{k}^{++}$) subset $C \in V$ (the proof of [13, Lemma 22.25] works in this case as well), and hence $S_{k}^{0}$ as well as $\rho_{k}^{++} \backslash S_{k}^{0}$ are $\rho_{k}^{+}$-stationary subsets of $\rho_{k}^{++}$in $V\left[G_{k+1}\right]$. The rest of our forcing is $\rho_{k}^{+++}$-closed, and hence $S_{k}^{0}$ and $\rho_{k}^{++} \backslash S_{k}^{0}$ remain $\rho_{k}^{+}$-stationary in $V[G * g]$. Let $S^{0}$ be such that $g$ is the characteristic function of $S^{0}$ and $S^{1}=\kappa^{++} \backslash S^{0}$. Again, $S^{0}$ and $S^{1}$ are $\kappa^{+}$-stationary subsets of $\kappa^{++}$ in $V[G * g]$.

$j(\mathbb{R})$ is the iteration with reverse Easton supports of length $j(\kappa)+1$. A standard argument gives $j(\mathbb{R})_{\kappa}=\mathbb{R}_{\kappa}$, and hence $G$ is $j(\mathbb{R})_{\kappa}$-generic over $M$ and $\left(H(\kappa)^{++}\right)^{V[G]}=\left(H(\kappa)^{++}\right)^{M[G]}$ (see [7, Lemma 4.4]). From the above it follows that $F n\left(\kappa^{++}, 2, \kappa^{++}\right)^{V[G]}=F n\left(\kappa^{++}, 2, \kappa^{++}\right)^{M[G]}$, and therefore $\mathbb{R}=j(\mathbb{R})_{\kappa+1}$ and $g$ is $F n\left(\kappa^{++}, 2, \kappa^{++}\right)$-generic over $M$ as well.

Suppose that there exists a $j(\mathbb{R})$-generic filter $G^{\prime}=G * g * H * h \in$ $V[G * g]$ over $M$ such that $H$ is a $j(\mathbb{R})_{\kappa, j(\kappa)}$-generic over $M[G * g], h$ is $\mathbb{S}_{j(\kappa)}=j\left(\dot{\mathbb{S}}_{\kappa}\right)^{G * g * H}$-generic over $M[G * g * H]$, and $j[G * g] \subset G^{\prime}$. Then $j$ can be extended to an elementary embedding $j^{\prime}: V[G * g] \rightarrow M\left[G^{\prime}\right]$ such that $j^{\prime}(G * g)=G^{\prime}$ (see [6, Proposition 9.1]). Therefore $j^{\prime}\left\langle S_{k}^{0}: k \in \kappa\right\rangle(\kappa)=S^{0}$.

$\left({ }^{3}\right)$ We could assume here that the domain of $f$ is still $H(\kappa)^{V}$ and $a \in H\left(\kappa^{++}\right)^{V}$, but this is irrelevant. 
In addition, conditions (i) and (ii) hold by [6, Proposition 9.3]. Thus $j^{\prime}$, $V^{\prime}=V[G * g]$, and $M^{\prime}=M[H]$ are as required.

It suffices to note that such $H$ and $h$ exist: the construction of $H$ is standard, see, e.g., fourth, fifth and sixth paragraphs of the proof of 7 , Theorem 4.2]; the existence of $h$ follows from the $\kappa^{+}$-distributivity of $\mathbb{S}_{\kappa}$ by virtue of [6, Proposition 15.1], which implies that the subfilter $h$ of $\mathbb{S}_{j(\kappa)}$ generated by $j[g]$ is as required.

There is no loss of generality in assuming $j=j^{\prime}, V=V^{\prime}$, and $M=M^{\prime}$. We define a forcing poset $\mathbb{P}$ as follows. Let $\mathbb{P}_{0}=\left\{\mathbf{1}_{0}\right\}$. For $k \leq \kappa$ we denote by $\dot{\mathbb{Q}}_{k}$ a $\mathbb{P}_{k}$-name for $S T_{S_{k}^{0}, S_{k}^{1}}\left({ }^{4}\right)$. Proceeding this way along all inaccessible cardinals $\leq \kappa$ and using reverse Easton supports we define $\mathbb{P}$. Observe that $\mathbb{P}_{k}$ has $\rho_{k}^{+}$-c.c., and hence $S_{k}^{0}, S_{k}^{1}$ are still $\rho_{k}^{+}$-stationary in $V^{\mathbb{P}_{k}}$. From the above and Theorem 4.10 we see that $V^{\mathbb{P}} \vDash \operatorname{cf}(\operatorname{Sym}(\kappa))=\kappa^{++}$. Thus it suffices to prove that $\kappa$ is measurable in $V^{\mathbb{P}}$. In order to do this we shall extend $j$ to an elementary embedding from $V^{\mathbb{P}}$ into $M^{j(\mathbb{P})}$.

$j(\mathbb{P})$ is an iteration of length $j(\kappa)+1$ in $M$ with reverse Easton support. It is clear that $j(\mathbb{P})_{\kappa}=\mathbb{P}_{\kappa}$. Let $G$ be a $\mathbb{P}_{\kappa}$-generic filter over $V$. Since $M$ and $V$ have the same $H\left(\kappa^{++}\right)$and $j\left\langle\left(S_{k}^{0}, S_{k}^{1}\right): k \in \kappa\right\rangle(\kappa)=\left(S^{0}, S^{1}\right)$, we have $\left(\kappa^{++}\right)^{M[G]}=\left(\kappa^{++}\right)^{V[G]}$ (see [7, Lemma 4.4]) and $j(\mathbb{P})_{\kappa+1}=\mathbb{P}$. Note that $j(\mathbb{P})=j\left(\mathbb{P}_{\kappa}\right) * j\left(\dot{\mathbb{Q}}_{\kappa}\right)$. Let $g$ be generic for $\dot{\mathbb{Q}}_{\kappa}^{G}$ over $V[G]$. We need to find a suitable $j(\mathbb{P})$-generic filter over $M$ in order to lift $j$ to $V[G * g]$. The following claim is analogous to [1, Lemma 6.4].

Claim 5.2. If $x \subset M[G]$ (resp. $x \subset M[G * g]$ ), $x \in V[G]$ (resp. $x \in$ $V[G * g])$, and $V[G] \vDash|x| \leq \kappa($ resp. $V[G * g] \vDash|x| \leq \kappa)$, then $x \in M[G]$ (resp. $x \in M[G * g]$ ).

Proof. We present the proof of the $G * g$ part only. The other part is analogous. Without loss of generality, $x$ is a set of ordinals. Let $\dot{x}$ be a $\mathbb{P}$-name such that $\dot{x}^{G * g}=x$. The $\kappa^{++}$-c.c. of $\mathbb{P}$ yields a set of ordinals $y \in V$ of size $|y| \leq \kappa^{+}$in $V$ and a condition $q \in \mathbb{P}$ such that $q \Vdash \dot{x} \subset y$. For every $\alpha \in y$ there exists a maximal (in $\{p \in \mathbb{P}: p \leq q\}$ ) antichain $A_{\alpha}$ of conditions $p$ such that $p \Vdash \alpha \in \dot{x}$ for every $p \in A_{\alpha}$. Applying Theorem 2.9, we conclude that $\left|A_{\alpha}\right| \leq \kappa^{+}$for every $\alpha \in y$. It is clear that $\left\langle A_{\alpha}: \alpha \in y\right\rangle \in H\left(\kappa^{++}\right)$, and hence $\left\langle A_{\alpha}: \alpha \in y\right\rangle \in\left(H\left(\kappa^{++}\right)\right)^{M}$. It suffices to note that $x=\{\alpha \in y$ : $\left.G * g \cap A_{\alpha} \neq \emptyset\right\}$.

In the same way as in the proof of [7, Theorem 4.2] (using Claim 5.2 instead of [11, Lemma 3]) we can find a $j(\mathbb{P})\lceil(\kappa, j(\kappa))$-generic filter $H \in$ $V[G * g]$ over $M[G * g]$. Thus $j[G]=G \subset G * g * H$, and hence $j$ lifts to an embedding $j^{*}: V[G] \rightarrow M[G * g * H]$ definable in $V[G * g]$ (see [6, Proposition 9.1]). Let $M^{*}$ denote $M[G * g * H]$.

$\left({ }^{4}\right)$ Here $S_{\kappa}^{0}=S^{0}$ and $S_{\kappa}^{1}=S^{1}$. 
We give Definition 5.3 and Claim 5.4 in full generality for any iteration of Miller and Sacks forcings.

DEFINITION 5.3. Let $\rho$ be a strongly inaccessible cardinal and $\gamma$ be an ordinal, $S^{0}, S^{1}$ be disjoint sets such that $S^{0} \cup S^{1}=\gamma$, and $\vec{A}=\left\langle A_{\alpha}\right.$ : $\alpha<\rho\rangle$ be a sequence of elements of $\rho$. Suppose that $\left\langle\left(p_{\alpha}, F_{\alpha}\right): \alpha \in \rho\right\rangle$ is a generalized fusion sequence for $S T_{S^{0}, S^{1}, \vec{A}}, q=\bigwedge_{\alpha<\rho} p_{\alpha}$, and $i \in \rho$. We say that a function $\sigma: F \rightarrow \rho^{i+1}$ is i-properly situated on $q$ (with respect to the fusion sequence $\left.\left\langle\left(p_{\alpha}, F_{\alpha}\right): \alpha \in \rho\right\rangle\right)$ if $F_{i} \subset F, \sigma$ lies on some $r \leq q$ such that $r|\xi \Vdash \sigma(\xi)| i \in \max _{\operatorname{Split}_{i}}(q(\xi))$ for all $\xi \in F$, and $\sigma(\xi)(i)=i$ for all $\xi \in F \cap S^{0}$.

Claim 5.4. Let $\rho, S^{0}, S^{1}, \vec{A},\left\langle\left(p_{\alpha}, F_{\alpha}\right): \alpha \in \rho\right\rangle, q, i$ be as in Definition 5.3, $u \leq q, F, T \in[\gamma]^{<\rho}$ with $F \subset T$, and $C \subset \rho$ be a club. Then there exists $v \leq_{F, i} u$ satisfying the following conditions:

For every $\sigma: F \rightarrow \rho^{i+1}$ which lies on $v$ and has the property $\sigma(\xi)(i)=i$ for all $\xi \in F \cap S^{0}$, there exist $j \in C$ and $\pi: T \cup F_{j} \rightarrow \rho^{(j+1)}$ such that $\pi(\xi)\lceil(i+1)=\sigma(\xi)$ for all $\xi \in F, \pi$ lies on $v, v|\sigma=v| \pi$, and $v \mid \pi$ is a witness for $\pi$ being $j$-properly situated on $q$ with respect to $\left\langle\left(p_{\alpha}, F_{\alpha}\right): \alpha \in \rho\right\rangle$.

Proof. Let us enumerate as $\left\{\sigma^{\zeta}: \zeta \in \eta\right\}$ all $\sigma: F \rightarrow \rho^{i+1}$ with the property $\sigma(\xi)(i)=i$ for all $\xi \in F \cap S^{0}$ and which lie on some $r \leq u$. Set $u_{0}=u$ and suppose that for some $\zeta<\eta$ and all $\zeta^{\prime}<\zeta$ we have already defined $u_{\zeta^{\prime}} \in S T_{S^{0}, S^{1}, \vec{A}}$ such that $u_{\zeta^{\prime}} \leq_{F, i} u_{\zeta^{\prime \prime}}$ for all $\zeta^{\prime \prime} \leq \zeta^{\prime}<\zeta$. If $\zeta$ is limit, we set $u_{\zeta}=\bigwedge_{\zeta^{\prime} \in \zeta} u_{\zeta^{\prime}}$.

Let us consider the case $\zeta=\zeta^{\prime}+1$. If there is no $r \leq u_{\zeta^{\prime}}$ such that $\sigma^{\zeta^{\prime}}$ lies on $r=r \mid \sigma^{\zeta^{\prime}}$, then we set $u_{\zeta}=u_{\zeta^{\prime}}$. Otherwise set $r_{0}^{\zeta^{\prime}}=r, \sigma_{0}^{\zeta^{\prime}}=\sigma^{\zeta^{\prime}}$, and $F_{\alpha}^{\zeta^{\prime}}=F_{\alpha} \cup T$. Repeating the same argument as in Claim 2.11, we can construct a sequence $\left\langle r_{\alpha}^{\xi^{\prime}}: \alpha \in \rho\right\rangle$ of elements of $S T_{S^{0}, S^{1}, \vec{A}}$, a sequence $\left\langle\sigma_{\alpha}^{\zeta^{\prime}}: F_{\alpha}^{\zeta^{\prime}} \rightarrow \rho^{<\rho} \mid \alpha<\rho\right\rangle$, and sequences $\left\langle\mu_{\alpha, \xi}^{\zeta^{\prime}}, \nu_{\alpha, \xi}^{\zeta^{\prime}}: \alpha \in \rho, \xi \in F_{\alpha}^{\zeta^{\prime}}\right\rangle$ of ordinals less than $\rho$ satisfying (i) $-(\mathrm{v})$ of Claim 2.11. Claim 2.12 yields a club $C^{\zeta^{\prime}} \subset \rho$ such that $\mu_{\alpha, \xi}^{\zeta^{\prime}}=\nu_{\alpha, \xi}^{\zeta^{\prime}}=\alpha$ and $\sigma_{\alpha}^{\zeta^{\prime}}(\xi)\left(\mu_{\alpha, \xi}^{\zeta^{\prime}}\right)=\alpha$ for every $\alpha \in C^{\zeta^{\prime}}$ and $\xi \in F_{\alpha}^{\zeta^{\prime}} \cap S^{0}$. Let us fix $j^{\zeta^{\prime}} \in C^{\zeta^{\prime}} \cap C$ and set $\pi^{\zeta^{\prime}}=\sigma_{j \zeta^{\prime}}^{\zeta^{\prime}}$ and $r^{\zeta^{\prime}}=r_{j \zeta^{\prime}+1}^{\zeta^{\prime}}$. By Claim 2.11(iii),(iv) we have $r^{\zeta^{\prime}} \mid \pi^{\zeta^{\prime}}=r^{\zeta^{\prime}}$ and $r^{\zeta^{\prime}}$ is a witness for $\pi^{\zeta^{\prime}}$ being $j^{\zeta^{\prime}}$-properly situated on $q$. Now let $u_{\zeta}$ be the amalgamation of $u_{\zeta^{\prime}}$ and $r \zeta^{\prime}$ defined as follows:

(a) $\operatorname{supp}\left(u_{\zeta}\right)=\operatorname{supp}\left(r^{\zeta^{\prime}}\right)$.

(b) If $\xi \in F$, then $u_{\zeta}(\xi)$ is such that

$$
r^{\zeta^{\prime}} \mid \xi \Vdash u_{\zeta}(\xi)=\left(u_{\zeta^{\prime}}(\xi) \backslash u_{\zeta^{\prime}}(\xi)_{\sigma^{\zeta^{\prime}}(\xi)}\right) \cup r^{\zeta^{\prime}}(\xi),
$$


and for any condition $c \leq u_{\zeta}\left\lceil\xi\right.$ incompatible with $r^{\zeta^{\prime}} \mid \xi, c \Vdash_{\xi} u_{\zeta}(\xi)=$ $u_{\zeta^{\prime}}(\xi)$.

(c) If $\xi \notin F$, then $u_{\zeta}(\xi)$ is such that $r^{\zeta^{\prime}} \mid \xi \Vdash u_{\zeta}(\xi)=r^{\zeta^{\prime}}(\xi)$, and for any condition $c \leq u_{\zeta}\left\lceil\xi\right.$ incompatible with $r^{\zeta^{\prime}}\left\lceil\xi, c \Vdash_{\xi} u_{\zeta}(\xi)=u_{\zeta^{\prime}}(\xi)\right.$.

By the definition of $u_{\zeta}$ we have

$$
u_{\zeta}\left|\sigma^{\zeta^{\prime}}=r^{\zeta^{\prime}}=r^{\zeta^{\prime}}\right| \pi^{\zeta^{\prime}}=\left(u_{\zeta} \mid \sigma^{\zeta^{\prime}}\right)\left|\pi^{\zeta^{\prime}}=u_{\zeta}\right| \pi^{\zeta^{\prime}}
$$

and $u_{\zeta} \leq_{F, i} u_{\zeta^{\prime}}$.

We claim that $v=\bigwedge_{\zeta<\eta} u_{\zeta}$ is as required. Indeed, let $\sigma: F \rightarrow \rho^{i+1}$ be as in the formulation. Since $v \leq u$, we have $\sigma=\sigma^{\zeta}$ for some $\zeta \in \eta$ and the construction of $u_{\zeta+1}$ is nontrivial. From the above it follows that $v\left|\sigma \leq u_{\zeta}\right| \sigma^{\zeta}=u_{\zeta} \mid \pi^{\zeta}$, consequently $\pi^{\zeta}$ lies on $v$ and $v|\sigma=v| \pi^{\zeta} \leq u_{\zeta} \mid \pi^{\zeta}=r^{\zeta}$. Now it is easy to see that $j=j^{\zeta}$ and $\pi=\pi^{\zeta}$ are as required.

Claim 5.5. Let $\rho, S^{0}, S^{1}$, and $\vec{A}$ be as in Definition 5.3, and $p \in$ $S T_{S^{0}, S^{1}, \vec{A}}$. Then for every sequence $\left\langle D_{\alpha}: \alpha \in \rho\right\rangle$ of open dense subsets of $S T_{S^{0}, S^{1}, \vec{A}}$ there exists a generalized fusion sequence $\left\langle\left(p_{\alpha}, F_{\alpha}\right): \alpha \in \rho\right\rangle$ with $p_{0}=p$ and such that, with $q=\bigwedge_{\alpha \in \rho} p_{\alpha}$, for every limit $i \in \rho$ and $\sigma: F_{i} \rightarrow \rho^{i+1}$ which is $i$-properly situated on $q$, $\sigma$ lies on $q$ and $q \mid \sigma \in D_{i}$.

Proof. Take $r_{\alpha, j} \in D_{\alpha}$ in the construction of a fusion sequence from the proof of Lemma 2.10 (the part before Claim 2.11) instead of demanding that $r_{\alpha, j}$ decides $\dot{z}$ as a ground model object. The resulting fusion sequence is easily seen to be as required.

Let us come back to our main task, namely to extend $j^{*}$ to an elementary embedding $j^{* *}: V[G * g] \rightarrow M^{*}[h]$ for some $\mathbb{Q}_{j(\kappa)}:=j^{*}\left(\dot{\mathbb{Q}}_{\kappa}^{G}\right)=$

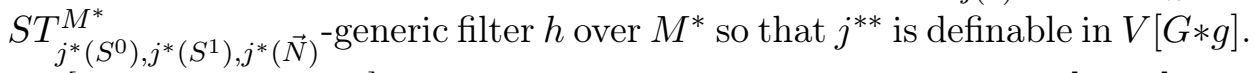
By [6. Proposition 9.1] it is enough to find a $\mathbb{Q}_{j(\kappa)}$-generic $h \in V[G * g]$ over $M^{*}$ for which $j^{*}[g] \subset h$.

For every $\xi<\kappa^{++}$we denote by $x(\xi) \in \kappa^{\kappa} \cap V[G * g]$ the (unique!) branch through all trees in $g(\xi)$ and let $a_{\xi}=\kappa$ (resp. $a_{\xi}=0$ ) for all $\xi \in S^{0}$ (resp. $\left.\xi \in S^{1}\right)$. We claim that

$$
h=\left\{j^{*}(p)\left|\sigma_{I}: p \in g, I \in M^{*}, I \subset j\left[\kappa^{++}\right],\right| I \mid=\kappa\right\},
$$

where $\sigma_{I}(j(\xi))=x(\xi)^{\wedge} a_{\xi}$ for all $j(\xi) \in I$, is $\mathbb{Q}_{j(\kappa)}$-generic over $M^{*}$. The proof below is a generalization of the "tuning fork" argument invented in [11. Let $\bar{D} \in M^{*}$ be an open dense subset of $\mathbb{Q}_{j(\kappa)}$. Write $\bar{D}$ as $j^{*}(f)(\bar{a})$, where $f$ has domain $H(\kappa)^{V}, f \in V[G]$, and $\bar{a} \in H\left(\kappa^{++}\right)^{V}$. There is no loss of generality to assume that $f(a)$ is open dense in $\mathbb{Q}_{\kappa}:=\dot{\mathbb{Q}}_{\kappa}^{G}$ for all $a \in H(\kappa)^{V}$. Let us enumerate $H(\kappa)^{V}$ as $\left\langle a_{k}: k \in \kappa\right\rangle$ and set $D_{k}=\bigcap_{k^{\prime} \leq k} f\left(a_{k^{\prime}}\right)$. 
Let $p \in \mathbb{Q}_{\kappa}$ be arbitrary. Claim 5.5 yields a generalized fusion sequence $\left\langle\left(p_{k}, F_{k}\right): k \in \kappa\right\rangle$ such that $p_{0}=p$ and, with $q=\bigwedge_{k \in \kappa} p_{k}$, for every limit $k \in \kappa$ and $\sigma$ which is $k$-properly situated on $q, \sigma$ lies on $q$ and $q \mid \sigma \in D_{k}$.

Let $\left\langle\bar{F}_{\bar{k}}: \bar{k} \in j(\kappa)\right\rangle$ and $\left\langle\bar{p}_{\bar{k}}: \bar{k} \in j(\kappa)\right\rangle$ be the results of applying $j^{*}$ to $\left\langle F_{k}: k \in \kappa\right\rangle$ and $\left\langle p_{k}: k \in \kappa\right\rangle$ respectively. By elementarity of $j^{*},\left\langle\left(\bar{p}_{\bar{k}}, \bar{F}_{\bar{k}}\right)\right.$ : $\bar{k} \in j(\kappa)\rangle$ is a generalized fusion sequence for $\mathbb{Q}_{j(\kappa)}, \bar{q}:=j^{*}(q)=\bigwedge_{\bar{k}<j(\kappa)} \bar{p}_{\bar{k}}$, and there exists $\bar{\beta} \in j(\kappa)$ so that for each limit $\bar{\alpha} \geq \bar{\beta}$ and $\bar{\sigma}$ which is $\bar{\alpha}$-properly situated on $\bar{q}, \bar{\sigma}$ lies on $\bar{q}$ and $\bar{q} \mid \bar{\sigma} \in \bar{D}$. We can additionally assume that $\bar{\beta}>\kappa$.

Fix $u \leq q$ and a club $C \subset \kappa$ such that $j(C) \cap(\kappa, \bar{\beta}]=\emptyset$ (its existence is established, e.g., in the proof of [11, Lemma 4]). Using Claim 5.4, we can construct a fusion sequence $\left\langle\left(u_{k}, T_{k}\right): k \in \kappa\right\rangle$ with $u_{0}=u$ satisfying the following conditions:

(i) $F_{k} \subset T_{k}$.

(ii) For every $\sigma: T_{k} \rightarrow \kappa^{k+1}$ which lies on $u_{k}$ and has the property $\sigma(\xi)(k)=k$ for all $\xi \in T_{k} \cap S^{0}$, there exist a limit ordinal $m \in$ $C \backslash(k+1)$ and $\pi: T_{k+1} \cup F_{m} \rightarrow \kappa^{(m+1)}$ such that $\pi(\xi)\lceil(k+1)=\sigma(\xi)$ for all $\xi \in T_{k}, \pi$ lies on $u_{k+1}, u_{k+1}\left|\sigma=u_{k+1}\right| \pi$, and $u_{k+1} \mid \pi$ is a witness for $\pi$ being $m$-properly situated on $q$ with respect to $\left\langle\left(p_{k}, F_{k}\right): k \in \kappa\right\rangle$.

Let $\left\langle\bar{T}_{\bar{k}}: \bar{k} \in j(\kappa)\right\rangle$ and $\left\langle\bar{u}_{\bar{k}}: \bar{k} \in j(\kappa)\right\rangle$ be the results of applying $j^{*}$ to $\left\langle T_{k}: k \in \kappa\right\rangle$ and $\left\langle u_{k}: k \in \kappa\right\rangle$ respectively, $v=\bigwedge_{k<\kappa} u_{k}$, and $\bar{v}=j^{*}(v)=\bigwedge_{\bar{k}<j(\kappa)} \bar{u}_{\bar{k}}$. By elementarity of $j^{*}$, for every $\bar{\sigma}: \bar{T}_{\kappa} \rightarrow j(\kappa)^{\kappa+1}$ which lies on $\bar{u}_{\kappa}$ and has the property $\bar{\sigma}(\bar{\xi})(\kappa)=\kappa$ for all $\bar{\xi} \in \bar{T}_{\kappa} \cap j\left(S^{0}\right)$, there exist a limit ordinal $\bar{m} \in j(C) \backslash(\kappa+1)$ and $\bar{\pi}: \bar{T}_{\kappa+1} \cup \bar{F}_{\bar{m}} \rightarrow j(\kappa)^{(\bar{m}+1)}$ such that $\bar{\pi}(\bar{\xi})\left\lceil(\kappa+1)=\bar{\sigma}(\bar{\xi})\right.$ for all $\bar{\xi} \in \bar{T}_{\kappa}, \bar{\pi}$ lies on $\bar{u}_{\kappa+1}, \bar{u}_{\kappa+1}\left|\bar{\sigma}=\bar{u}_{\kappa+1}\right| \bar{\pi}$, and $\bar{u}_{\kappa+1} \mid \bar{\sigma}$ is a witness for $\bar{\pi}$ being $\bar{m}$-properly situated on $\bar{q}$ with respect to $\left\langle\left(\bar{p}_{\bar{k}}, \bar{F}_{\bar{k}}\right): \bar{k} \in j(\kappa)\right\rangle$.

Since $p$ and $u \leq q$ were chosen arbitrarily, we can assume that $v \in g$. Observe that $\bar{T}_{\kappa}=\bigcup_{k \in \kappa} j\left[T_{k}\right] \subset j\left[\kappa^{++}\right],\left|\bar{T}_{\kappa}\right|=\kappa$, and $\bar{T}_{\kappa} \in M^{*}$. The elementarity of $j^{*}$ implies that $\bar{\sigma}:=\sigma_{\bar{T}_{\kappa}}$ lies on $j^{*}(w)$ for any $w \in g$. In particular, $\bar{\sigma}$ lies on $\bar{u}_{k}=j^{*}\left(u_{k}\right)$ for all $k \in \kappa$, and hence it lies on $\bar{u}_{\kappa}=\bigwedge_{k \in \kappa} \bar{u}_{k}$ as well. Therefore we can find $\bar{m} \in j(C) \backslash(\kappa+1)$ and $\bar{\pi}: \bar{T}_{\kappa+1} \cup \bar{F}_{\bar{m}} \rightarrow j(\kappa)^{(\bar{m}+1)}$ as above, i.e. $\bar{u}_{\kappa+1} \mid \bar{\sigma}$ is a witness for $\bar{\pi}$ being $\bar{m}$-properly situated on $\bar{q}$ with respect to $\left\langle\left(\bar{p}_{\bar{k}}, \bar{F}_{\bar{k}}\right): \bar{k} \in j(\kappa)\right\rangle$. By the construction of $\left\langle\left(p_{k}, F_{k}\right): k \in \kappa\right\rangle$, elementarity of $j^{*}$, the equalities $j(C) \cap(\kappa, \bar{\beta})=\emptyset$ and $\bar{m} \in j(C) \backslash(\kappa+1)$, and our choice of $\bar{\beta}$, we conclude that $\bar{\pi} \mid \bar{F}_{\bar{m}}$ lies on $\bar{q}$ and $\bar{q} \mid\left(\bar{\pi} \mid \bar{F}_{\bar{m}}\right) \in \bar{D}$. On the other hand, 


$$
\bar{q}\left|\left(\bar{\pi} \mid \bar{F}_{\bar{m}}\right) \geq \bar{u}_{\kappa+1}\right| \bar{\pi}=\bar{u}_{\kappa+1}|\bar{\sigma} \geq \bar{v}| \bar{\sigma}=j^{*}(v) \mid \sigma_{\bar{T}_{\kappa}} \in h,
$$

which means that $h \cap \bar{D} \neq \emptyset$ and thus finishes the proof of Theorem 1.1.

REMARK 5.6. 1. To the best knowledge of the authors there are essentially three other different forcing extensions $V^{P}$ of $V$ which preserve the measurability of $\kappa$ and kill the GCH at $\kappa$ under the assumption that $\kappa$ is $P_{2} \kappa$-hypermeasurable (see [6, §24], [11], and [7, §4]). In all three cases we have $\operatorname{cf}(\operatorname{Sym}(\kappa))=\kappa^{+}$in $V^{P}$. The historically first of them is due to Woodin [6, $\S 24]$. His $P$ can be written as $P_{0} * P_{1} * P_{2}$, where $P_{0}$ is iteration of Cohen posets below $\kappa$ with reverse Easton support, and thus $\left|P_{0}\right|=\kappa$ and $P_{0}$ has $\kappa$-c.c.; $P_{1}$ is the poset adding $\kappa^{++}$-many Cohen subsets of $\kappa$, and $P_{2}$ adds no new subsets of $\kappa$. It is clear that $V^{P_{0} * P_{1}} \vDash \operatorname{cf}(\operatorname{Sym}(\kappa))=\kappa^{+}$(see the last paragraph in [22, p. 894]), and a forcing which does not add new subsets of $\kappa$ cannot enlarge $\operatorname{cf}(\operatorname{Sym}(\kappa))$.

In forcing extensions constructed in [1] and [7] the equality $\mathfrak{d}(\kappa)=\kappa^{+}$ holds, and it is well-known (the proof of [21, Proposition 1.4] works for every regular $\kappa)$ that $\operatorname{cf}(\operatorname{Sym}(\kappa)) \leq \mathfrak{d}(\kappa)$ for every regular $\kappa$.

2. It is known 22 that the equality $\operatorname{cf}(\operatorname{Sym}(\kappa))=\kappa^{++}$(and much more) is consistent for every inaccessible $\kappa$. But the authors were not able to lift elementary embeddings to forcing extensions used in $[22]\left({ }^{5}\right)$ assuming considerably less than supercompactness. However, such a possibility is not formally excluded. On the other hand, applying the methods developed in [10] to forcing extensions from [22] we could obtain the following result:

Suppose $0^{\sharp}$ exists. Then there is an inner model in which $\operatorname{cf}(\operatorname{Sym}(\kappa))=$ $\kappa^{++}$for every regular cardinal $\kappa$ of the form $\aleph_{2 \alpha}$.

It is worth mentioning here that for every cardinal $\kappa$ the inequality $\operatorname{cf}(\operatorname{Sym}(\kappa))>\kappa^{+}$implies $\operatorname{cf}\left(\operatorname{Sym}\left(\kappa^{+}\right)\right) \leq \operatorname{cf}(\operatorname{Sym}(\kappa))$, and it is not known even how to obtain $\operatorname{cf}(\operatorname{Sym}(\kappa))>\kappa^{+}$at two consecutive $\kappa$ simultaneously (see [22]).

3. In order to show that $j(\mathbb{P})_{\kappa+1}=\mathbb{P}$ in the proof of Theorem 1.1 we needed suitable stationary sets $S^{0}, S^{1}$ and $\left\langle S_{k}^{0}, S_{k}^{1}: k \in \kappa\right\rangle$. Instead of using the auxiliary forcing introducing such sets we could apply the same inner model argument as in the proof of [9, Theorem 11].

Acknowledgments. We thank the anonymous referee for a careful reading and many suggestions which improved the paper.

We also thank FWF grant P19898-N18 for support for this research.

$\left({ }^{5}\right)$ The forcing posets used in [22] were developed in [17, $\left.§ 2,3\right]$. 


\section{References}

[1] J. E. Baumgartner, Iterated forcing, in: Surveys in Set Theory, A. R. D. Mathias (ed.), London Math. Soc. Lecture Note Ser. 87, Cambridge Univ. Press, Cambridge, 1983, 1-59.

[2] A. Blass and C. Laflamme, Consistency results about filters and the number of inequivalent growth types, J. Symbolic Logic 54 (1989), 50-56.

[3] A. Blass and S. Shelah, There may be simple $P_{\aleph_{1}}-$ and $P_{\aleph_{2}}$-points, and the RudinKeisler ordering may be downward directed, Ann. Pure Appl. Logic 33 (1987), 213243.

[4] J. Brendle and M. Losada, The cofinality of the infinite symmetric group and groupwise density, J. Symbolic Logic 68 (2003), 1354-1361.

[5] E. T. Brown and M. J. Groszek, Uncountable superperfect forcing and minimality, Ann. Pure Appl. Logic 144 (2006), 73-82.

[6] J. Cummings, Iterated forcing and elementary embeddings, in: Handbook of Set Theory, M. Foreman et al. (eds.), Kluwer, Dordrecht, to appear.

[7] N. Dobrinen and S.-D. Friedman, The tree property at the double successor of a measurable, preprint, 2008; http://www.logic.univie.ac.at/ ${ }^{\mathrm{sdf} / \text { papers/joint.natasha. }}$ tp.pdf

[8] T. Eisworth, On iterated forcing for successors of regular cardinals, Fund. Math. 179 (2003), 249-266.

[9] S.-D. Friedman and M. Magidor, The number of normal measures, J. Symbolic Logic 74 (2009), 1069-1080.

[10] S.-D. Friedman and K. Thompson, Internal consistency for embedding complexity, ibid. 73 (2008), 831-844.

[11] —, -, Perfect trees and elementary embeddings, ibid. 73 (2008), 906-918.

[12] M. Gitik, The negation of the singular cardinal hypothesis from $o(\kappa)=\kappa^{++}$, Ann. Pure Appl. Logic 43 (1989), 209-234.

[13] T. Jech, Set Theory, Springer, Berlin, 2003.

[14] A. Kanamori, Perfect-set forcing for uncountable cardinals, Ann. Math. Logic 19 (1980), 97-114.

[15] K. Kunen, Set Theory. An Introduction to Independence Proofs, Stud. Logic Found. Math. 102, North-Holland, Amsterdam, 1980.

[16] H. D. Macpherson and P. M. Neumann, Subgroups of infinite symmetric groups, J. London Math. Soc. (2) 42 (1990), 64-84.

[17] A. H. Mekler and S. Shelah, Uniformization principles, J. Symbolic Logic 54 (1989), 441-459.

[18] A. Miller, Rational perfect set forcing, in: Axiomatic Set Theory, J. Baumgartner et al. (eds.), Contemp. Math. 31, Amer. Math. Soc., Providence, RI, 1984, 143159 .

[19] A. Rosłanowski and S. Shelah, Sheva-Sheva-Sheva: large creatures, Israel J. Math. 159 (2007), 109-174.

[20] J. D. Sharp and S. Thomas, Uniformisation problems and the cofinality of the infinite symmetric group, Notre Dame J. Formal Logic 35 (1994), 328-345.

[21] - - - Unbounded families and the cofinality of infinite symmetric group, Arch. Math. Logic 34 (1995), 33-45.

[22] - - - Some questions concerning the cofinality of Sym $(\kappa)$, J. Symbolic Logic 60 (1995), 892-897. 
[23] S. Thomas, Groupwise density and the cofinality of the infinite symmetric group, Arch. Math. Logic 37 (1998), 483-493.

Sy-David Friedman, Lyubomyr Zdomskyy

Kurt Gödel Research Center for Mathematical Logic

University of Vienna

Währinger Strasse 25

A-1090 Wien, Austria

E-mail: sdf@logic.univie.ac.at, lzdomsky@logic.univie.ac.at

http://www.logic.univie.ac.at/ ${ }^{\sim}$ sdf//, http://www.logic.univie.ac.at/ lzdomsky/

Received 10 November 2008;

in revised form 22 September 2009 